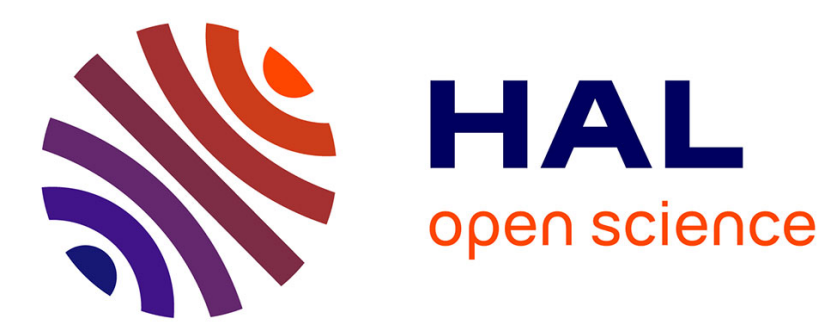

\title{
Analysis of waveform and amplitude of mouse rod and cone flash responses
}

Annia Abtout, Gordon Fain, Jürgen Reingruber

\section{To cite this version:}

Annia Abtout, Gordon Fain, Jürgen Reingruber. Analysis of waveform and amplitude of mouse rod and cone flash responses. The Journal of Physiology, 2021, 599 (13), pp.3295-3312. 10.1113/JP281225 . hal-03411942

\section{HAL Id: hal-03411942 \\ https://hal.science/hal-03411942}

Submitted on 4 Nov 2021

HAL is a multi-disciplinary open access archive for the deposit and dissemination of scientific research documents, whether they are published or not. The documents may come from teaching and research institutions in France or abroad, or from public or private research centers.
L'archive ouverte pluridisciplinaire HAL, est destinée au dépôt et à la diffusion de documents scientifiques de niveau recherche, publiés ou non, émanant des établissements d'enseignement et de recherche français ou étrangers, des laboratoires publics ou privés. 


\title{
Analysis of waveform and amplitude of mouse rod and cone flash responses
}

\author{
Annia Abtout ${ }^{1}$, Gordon Fain ${ }^{2,3}$ and Jürgen Reingruber ${ }^{1}$
}

${ }^{1}$ Institut de Biologie de l'École Normale Supérieure, 46 rue d'Ulm, 75005 Paris, France

${ }^{2}$ Department of Integrative Biology and Physiology, University of California, Los Angeles, CA 90095-7239, USA

${ }^{3}$ Department of Ophthalmology and Jules Stein Eye Institute, University of California, Los Angeles, CA 90095-7000, USA

Corresponding author:

Jürgen Reingruber

INSERM U1024

Institute of Biology of École Normale Supérieure

46 Rue d'Ulm

75005 Paris, France

Telephone: 0033-1-44-32-36-62

Email: reingrub@bio.ens.psl.eu

ORCID ID: 0000-0002-7167-8092

Acknowledgements: We are grateful to Alapakkam Sampath for reading an earlier draft of the manuscript. This work was funded by a grant from Agence Nationale de Recherche (ANR) to JR, NIH EY001844 to GLF, an unrestricted grant from Research to Prevent Blindness USA to the UCLA Department of Ophthalmology, and NEI Core Grant EY003311 to the Stein Eye Institute. 
The authors affirm that they have no competing interests. Reingruber designed the work. Abtout and Reingruber performed analysis. Reingruber and Fain wrote the manuscript.

\section{Key Points Summary}

- Most vertebrate eyes have rod and cone photoreceptors, which use a signal transduction pathway consisting of many biological processes to transform light into an electrical response.

- We dissect and quantify the contribution of each of these processes to the photoreceptor light response by using a novel method of analysis that provides an analytical solution for the entire time course of the dim-flash light response.

- We find that the shape of the light response is exclusively controlled by deactivation parameters. Activation parameters scale this shape and alter response amplitude.

- We show that the rising phase of the response depends on $\mathrm{Ca}^{2+}$ feedback, and we identify the deactivation parameters that control the recovery phase of the response.

- We devise new methods to extract values for deactivation and activation parameters from a separate analysis of response shape and response amplitude. 


\section{ABSTRACT}

Vertebrate eyes have rod and cone photoreceptors, which use a complex transduction pathway comprising many biological processes to transform the absorption of light into an electrical response. A fundamental question in sensory transduction is how these processes contribute to the response. To study this question, we use a well-accepted phototransduction model, which we analyze with a novel method based on the log-transform of the current. We derive an analytical solution that describes the entire time course of the photoreceptor response to dim flashes of light. We use this solution to dissect and quantify the contribution of each process to the response. We find that the entire dim-flash response is proportional to the flash intensity. By normalizing responses to unit amplitude, we define a waveform that is independent of the light intensity and characterizes the invariant shape of dim-flash responses. We show that this waveform is exclusively determined by deactivation rates; activation rates only scale the waveform and affect the amplitude. This analysis corrects a previous assumption that the rising phase is determined entirely by activation rates. We further show that the rising phase depends on $\mathrm{Ca}^{2+}$ feedback to the cyclase, contrary to current belief. We identify the deactivation rates that control the recovery phase of the response, and we devise new methods to extract activation and deactivation rates from an analysis of response shape and response amplitude. In summary, we provide a comprehensive understanding of how the various transduction processes produce the cellular response. 


\section{INTRODUCTION}

The signal transduction cascade of vertebrate rod and cone photoreceptors is a complex multistep process by which light-activation of a photopigment is transformed into a current response (see Burns \& Pugh, 2010; Arshavsky \& Burns, 2012; Reingruber et al., 2015; Fain, 2019). The photoreceptor response depends crucially on the interplay of two key molecules: the effector enzyme phosphodiesterase-6 (PDE), and the second messenger cyclic guanosine monophosphate (cGMP), which controls the opening of cyclic-nucleotide-gated (CNG) channels in the plasma membrane. Rhodopsin activation is coupled via a G-protein cascade to the activation of PDE, such that the number of activated PDE that are present in the outer segment at any time reflects the change in light intensity stimulating the photoreceptor. The light-signal encoded by the time course of light-activated PDE is then converted into a current response, because activated PDE hydrolyses cGMP and produces a net reduction in the number of open CNG channels. Finally, $\mathrm{Ca}^{2+}$-feedback modulates the transduction cascade, and this process is also light dependent because $\mathrm{Ca}^{2+}$ concentration is controlled by influx through $\mathrm{CNG}$ channels which close during the light response.

Experiments over the past 50 years have revealed many biological and biochemical details of this interconnected signaling pathway. More recently, considerable insight has come from experiments on genetically engineered photoreceptors, where specific components of the signaling cascade have been genetically modified. These findings have stimulated the development of sophisticated models, which we roughly classify into two main categories and which have different but complementary objectives. In the first category, modeling and mathematical analysis has been used to understand specific aspects of the photoreceptor response, for example (1) response amplification during the initial phase of the light response 
(Pugh \& Lamb, 1993, 2000), (2) dark noise and the variability of the single-photon response (Rieke \& Baylor, 1996; Rieke \& Baylor, 1998; Holcman \& Korenbrot, 2004; Reingruber \& Holcman, 2008a; Caruso et al., 2011; Reingruber et al., 2013), (3) spatio-temporal properties of cGMP diffusion and GMP hydrolysis by activated PDE (Cameron \& Pugh, 1990; Olson \& Pugh, 1993; Dumke et al., 1994; Koutalos \& Yau, 1996; Holcman \& Korenbrot, 2004; Caruso et al., 2006; Reingruber \& Holcman, 2008b; Gross et al., 2012b), or (4) analysis of $\mathrm{Ca}^{2+}$-feedback for light-adaptation and cGMP synthesis (Tranchina et al., 1991; Calvert et al., 1998; Burns et al., 2002; Chen et al., 2010c; Gross et al., 2012a). In the second category, highly complex models have been proposed to reproduce a large variety of experimental data including the effects of gene deletions and adaptation to background light (Field \& Rieke, 2002; Andreucci et al., 2003; Hamer et al., 2003; Hamer et al., 2005; Reingruber \& Holcman, 2008a; Shen et al., 2010; Korenbrot, 2012; Invergo et al., 2014; Astakhova et al., 2015; Lamb \& Kraft, 2020).

Most recent models have been of this second category and have contained many equations and parameters that can be adjusted by numerical fitting procedures. These models attempt to describe the photoreceptor response in all of its intricacy, but because of this large parameter space, it is difficult to infer from simulations and fittings how individual parameters or parameter combinations affect various properties of the light response. For example, the recovery phase of flash responses can be fit with a single exponential, but because there are many deactivation rates it is not obvious how the fitted exponential time constant relates to the various rates of the model. Another drawback of complex models is that considerable effort is required to implement them in order to use them for data analysis. This may explain why simple concepts like amplification constant, recovery time, and dominant time constant are still frequently applied to analyze data and to extract parameter values: the amplification constant introduced by 
Pugh and Lamb (1993) can be determined by fitting the initial phase of a flash response with a quadratic function, the recovery time constant by fitting the recovery phase with a single exponential, and the dominant time constant from a Pepperberg plot (Pepperberg et al., 1992).

A closer examination, however, reveals that the mathematical expressions or procedures that relate these concepts to underlying model parameters are not derived from a precise analysis of a phototransduction model. For example, the formula for the amplification constant has been derived from an analysis of amphibian rod responses without taking deactivation processes into account (Lamb \& Pugh, 1992), and this formula was then subsequently generalized to many other species (Pugh \& Lamb, 1993, 2000). It still remains unclear exactly how deactivation processes affect the rising phase. As another example, in WT rods recovery and dominant time constants are both identified with the PDE deactivation rate (Krispel et al., 2006; Tsang et al., 2006; Chen et al., 2010a), which is based on empirical observations of how these values change between phenotypes but lacks a theoretical basis.

To be able to perform a comprehensive mathematical analysis that provides a mathematical foundation for these widespread concepts, we have focused on a simple but representative model that comprises all the relevant transduction processes that are known to be necessary for a realistic flash response. We use a new mathematical approach based on the log transform of the current normalized by the amplitude of saturating flash responses to derive analytic expressions for the entire dim-flash response. We then apply these analytic results to verify the validity of asymptotic expressions, and to dissect the contributions of individual processes of the transduction pathway to the various phases of a flash response. In this way, we obtain a comprehensive understanding of how the variety of biochemical processes synergize to determine the final response. 


\section{METHODS}

\section{ANIMALS AND RECORDINGS}

The data we used to analyze photoreceptor responses came from experiments previously described (Ingram et al., 2019; Ingram et al., 2020; Reingruber et al., 2020). The recordings were taken from WT and GCAPs ${ }^{-/}$rods, and Gnat $^{-/}$and Gnat $^{-/-} ;$GCAPs $^{-/}$cones. GCAPs $^{-\sim}$ mutants lack the guanylyl-cyclase activating proteins (GCAPs), which are closely associated with guanylyl cyclase in both rods and cones and mediate $\mathrm{Ca}^{2+}$-dependent modulation of the rate of the cyclase. In $G C A P s^{-/}$mutants, $\mathrm{Ca}^{2+}$-dependent feedback to the cyclase is absent ( $\hat{\alpha}=1$, see below). Recordings from cones were made from Gnat $1^{-/ /}$retinas lacking Gnat1, the alpha-subunit of the rod $\mathrm{G}$ protein transducin. Cones in the mouse retina receive input from rods through connexin-36 gap junctions (Asteriti et al., 2017; Jin et al., 2020), and this input is deleted in Gnat $^{-/}$cones. There is no effect on cone transduction, however, with the result that Gnat $^{-/}$cones essentially behave like WT cones (Ingram et al., 2019). As we have previously explained (Reingruber et al., 2020), rod WT and $G C A P s^{-/-}$responses had different collecting areas because $G C A P s^{-/}$rod responses were recorded with suction electrodes, whereas WT rod (and cone) responses were recorded in retinal slices with voltage clamp. 


\section{MODEL}

We used a simplified phototransduction model that has been previously described (Reingruber et al., 2020). Except for the activation of transducin and the inclusion of $\mathrm{Ca}^{2+}$ feedback, this model is similar to that used by Pugh and Lamb to analyze the rising phase of the light response (Lamb \& Pugh, 1992; Pugh \& Lamb, 1993). In brief, the photoreceptor current $I$ as a function of the cyclic guanosine-nucleotide (cGMP) concentration $c_{c g}$ is approximated as $I=I_{d}\left(\frac{c_{c g}}{c_{c g, d}}\right)^{n_{c h}}$, where $I_{d}$ and $c_{c g, d}$ are the current and cGMP concentration in darkness, and $n_{c h}$ is the cooperativity constant of the channel. This approximation for the current assumes that the concentration of cGMP that half activates the channels is large in comparison to the basal cGMP concentration in darkness (Lamb \& Pugh, 1992; Pugh \& Lamb, 1993). The cGMP synthesis rate as a function of the $\mathrm{Ca}^{2+}$ concentration is $\alpha\left(c_{c a}\right)=\frac{\alpha_{\max }}{c_{c a}^{2}+K_{\alpha}^{2}}=\alpha_{d} \frac{c_{c a, d}^{2}+K_{\alpha}^{2}}{c_{c a}^{2}+K_{\alpha}^{2}}$, where $c_{c a, d}$ is the calcium concentration in darkness, $K_{\alpha}$ determines the sensitivity of the rate to $\mathrm{Ca}^{2+}$ (Tranchina et al., 1991; Burns et al., 2002; Hamer et al., 2003; Gross et al., 2012b; Lamb et al, 2018), $\alpha_{d}=$ $\beta_{d} c_{c g, d}$ is the synthesis rate in darkness that balances cGMP hydrolysis, and $\beta_{d}$ is the rate constant of cGMP hydrolysis in darkness (dark cGMP turnover rate).

For the PDE activation cascade, we assumed that light-activated visual pigment activates the G-protein transducin, which activates cGMP phosphodiesterase-6 (PDE). We let $R^{*}$ be the number of pigment molecules activated by the flash, $T^{*}$ the number of activated transducins, and $P^{*}$ the number of light-activated PDEs. The parameter $k_{a c t}$ is the rate of activation of transducin by a single activated visual pigment, $\mu_{r h}$ is the deactivation rate of a light-activated visual pigment molecule, and $\mu_{p d e}$ is the deactivation rate of a light-activated PDE. The activation rate 
$k_{t r}$ is the rate by which $T^{*}$ generates $P^{*}$, and $\mu_{t r}$ is the deactivation rate of $T^{*}$ (the rate of decrease of $T^{*}$ concentration). Because the generation of a $\mathrm{P}^{*}$ is accomplished by binding of $T^{*}$, we have $k_{t r}=\mu_{t r}$, such that a single $T^{*}$ generates a single light-activated PDE. The intermediate step by which $T^{*}$ activates PDE is often assumed to be fast and therefore omitted (Lamb \& Pugh, 1992; Pugh \& Lamb, 1993). We decided to keep this step as a representative reaction to study the effect of intermediate processes that delay the response without amplifying it. The value of $\mu_{t r}$ in Table 2 has been estimated in Reingruber et al. (2020) by fitting the model to the data. It therefore has to be considered as an effective value, which does not necessarily reflect the biological time scale by which $\mathrm{T}^{*}$ activates PDE.

With dimensionless quantities that are normalized with steady-state values in darkness, $\hat{c}_{c g}=\frac{c_{c g}}{c_{c g, d}}, \hat{c}_{c a}=\frac{c_{c a}}{c_{c a, d}}, \widehat{K}_{\alpha}=\frac{K_{\alpha}}{c_{c a, d}}, \hat{\alpha}=\frac{\alpha}{\alpha_{d}}=\frac{1+\widehat{K}_{\alpha}^{2}}{\hat{c}_{c a}^{2}+\widehat{K}_{\alpha}^{2}}$, and $\hat{I}=\frac{I}{I_{d}}=\hat{c}_{c g}{ }^{n_{c h}}$, the transduction equations are

$$
\begin{gathered}
\frac{d}{d t} R^{*}=\phi(t) \kappa-\mu_{r h} R^{*} \\
\frac{d}{d t} T^{*}=k_{a c t} R^{*}-\mu_{t r} T^{*} \\
\frac{d}{d t} P^{*}=k_{t r} T^{*}-\mu_{p d e} P^{*} \\
\frac{d}{d t} \hat{c}_{c g}=\beta_{d} \hat{\alpha}\left(\hat{c}_{c a}\right)-\left(\beta_{d}+\beta_{s u b} P^{*}\right) \hat{c}_{c g}
\end{gathered}
$$

where $\phi(t)$ is the light intensity and $\kappa$ is the collecting area. 
It remains to provide an equation for the $\mathrm{Ca}^{2+}$ concentration $\hat{c}_{c a}(t)$. As shown in Reingruber et al. (2013), the $\mathrm{Ca}^{2+}$ dynamics can be modelled as $\frac{d}{d t} \hat{c}_{c a}=\mu_{c a}\left(\hat{I}-\hat{c}_{c a}\right)$, where the rate constant $\mu_{c a}$ depends on the dark current, the outer segment volume, the buffering capacity $B_{c a}$, and the fraction of the CNG current that is carried by $\mathrm{Ca}^{2+}$ (see Eq. 33 in the SI of Reingruber et al., 2013). This equation further assumes that the half-saturating $\mathrm{Ca}^{2+}$ concentration $K_{e x}$ of the exchanger is large compared to the dark $\mathrm{Ca}^{2+}$ concentration $c_{c a, d}$ (Pugh $\&$ Lamb, 2000). With $B_{c a}=80$ we computed $\mu_{c a} \approx 23 s^{-1}$ for a mouse rod (Reingruber et al., 2013), and with a smaller buffering capacity $B_{c a} \sim 50$ (Gross et al., 2012a), we would get $\mu_{c a} \approx$ $37 s^{-1}$. For a mouse cone, due to the smaller volume, the value of $\mu_{c a}$ would be much higher.

Because $\mu_{c a}$ is large and not rate limiting, we simplify and use the approximation $\hat{c}_{c a}(t) \approx \hat{I}(t)$, such that the $\mathrm{Ca}^{2+}$ concentration changes proportional to the current. This approximation becomes exact in the limit $\mu_{c a} \rightarrow \infty$.

With the new variables $\tilde{P}^{*}=n_{c h} \beta_{s u b} P^{*}, \tilde{T}^{*}=\frac{n_{c h} \beta_{s u b} k_{t r}}{\mu_{p d e}} T^{*}, \tilde{R}^{*}=\frac{n_{c h} \beta_{s u b} k_{t r} k_{a c t}}{\mu_{p d e} \mu_{t r}} R^{*}, y=$ $-n_{c h} \ln \hat{c}_{c g}=-\ln \hat{I}$ and $\hat{c}_{c a}=e^{-y}$, the previous system of equations becomes

$$
\begin{gathered}
\frac{d}{d t} \tilde{R}^{*}=\mu_{r h}\left(\phi(t) \kappa \xi-\tilde{R}^{*}\right) \\
\frac{d}{d t} \tilde{T}^{*}=\mu_{t r}\left(\widetilde{R}^{*}-\tilde{T}^{*}\right) \\
\frac{d}{d t} \tilde{P}^{*}=\mu_{p d e}\left(\tilde{T}^{*}-\tilde{P}^{*}\right) \\
\frac{d}{d t} y=\tilde{P}^{*}-\beta_{d} H(y)
\end{gathered}
$$

where the gain $\xi$ is defined as 


$$
\xi=\frac{n_{c h} k_{a c t} \beta_{s u b} k_{t r}}{\mu_{r h} \mu_{p d e} \mu_{t r}}=\frac{n_{c h} k_{a c t} \beta_{s u b}}{\mu_{r h} \mu_{p d e}},
$$

and

$$
H(y)=n_{c h}\left(e^{\frac{y}{n_{c h}}} \hat{\alpha}\left(e^{-y}\right)-1\right)=n_{c h}\left(e^{\frac{y}{n_{c h}}} \frac{1+\widehat{K}_{\alpha}^{2}}{e^{-2 y}+\widehat{K}_{\alpha}^{2}}-1\right) .
$$

Eq. 1 shows that the activation rates affect the response only from the gain $\xi$ by means of the product $k_{a c t} \beta_{\text {sub }}$, which effectively modulates the flash intensity $\phi$. In this respect, $k_{a c t} \beta_{\text {sub }}$ behaves similarly to the collecting area $\kappa$ and could as well be incorporated into a modified collecting area $\tilde{\kappa}=\kappa k_{\text {act }} \beta_{\text {sub }}$. We have, however, elected to keep these variables separate for conceptional clarity. The parameter $n_{c h}$ cannot be treated in this way, because it further affects the value of the function $H(y)$ in Eq. 3 .

\section{Analytic results for PDE activation}

The equations for PDE activation are linear and can be solved analytically,

$$
\tilde{P}^{*}(t)=\kappa \xi \int_{0}^{t} \phi(s) g_{p}(t-s) d s
$$

with Green's function (also known as the impulse-response function) given by

$$
g_{p}(t)=\mu_{r h} \mu_{p d e} \mu_{t r}\left(\begin{array}{c}
\frac{e^{-\mu_{r h} t}}{\left(\mu_{r h}-\mu_{t r}\right)\left(\mu_{r h}-\mu_{p d e}\right)}+\frac{e^{-\mu_{t r} t}}{\left(\mu_{t r}-\mu_{r h}\right)\left(\mu_{t r}-\mu_{p d e}\right)}+ \\
+\frac{e^{-\mu_{p d e} t}}{\left(\mu_{p d e}-\mu_{t r}\right)\left(\mu_{p d e}-\mu_{r h}\right)}
\end{array}\right)
$$


The function $g_{p}(t)$ is symmetric in $\mu_{r h}, \mu_{t r}$ and $\mu_{p d e}$. For a flash at time $t=0$ with intensity $\phi$ and duration $\Delta t$, we have $\phi(t)=\phi(\theta(t)-\theta(t-\Delta t)$ ), where $\theta$ is the Heaviside (or unit step) function. If the flash duration $\Delta t$ is short and the flash produces $R_{0}^{*}=\kappa \phi \Delta t$ photoisomerizations, we get from Eq. 4 the approximation $\tilde{P}^{*}(t) \approx R_{0}^{*} \xi g_{p}(t)$.

\section{Analytic results for dim-flash responses}

It is not possible to solve Eq. 1 analytically with the non-linear function $H(y)$ from Eq. 3. For dim flashes where $y \ll 1$, however, we can approximate $H(y)$ by the leading-order term of its Taylor expansion. Because $H(y)=0$ for $y=0$, the leading order term is the linear expression $H(y)=y\left(1-n_{c h} \hat{\alpha}^{\prime}\right)$, where

$$
\hat{\alpha}^{\prime}=\left.\frac{d}{d \hat{c}_{c a}} \hat{\alpha}\left(\hat{c}_{c a}\right)\right|_{\hat{c}_{c a}=1}=-\frac{2}{1+\widehat{K}_{\alpha}^{2}}
$$

The parameter $\alpha^{\prime}$ describes how strongly the dark cyclase activity is modulated by $\mathrm{Ca}^{2+}$. For $\mathrm{GCAPs}^{-/}$mutants we have $\alpha^{\prime}=0$. With the parameters in Table 2, we compute for a WT rod $\alpha^{\prime} \approx-1.14$, and for a WT $\left(\right.$ Gnat1 $\left.^{-/}\right)$cone $\alpha^{\prime} \approx-1.17$, such that $1-n_{c h} \hat{\alpha}^{\prime} \approx 3.8$ for rod or cone photoreceptors. In the leading order approximation, we have $\frac{d}{d t} y=\widetilde{P}^{*}-\tilde{\beta}_{d} y$, where we have introduced the effective dark turnover rate

$$
\tilde{\beta}_{d}=\beta_{d}\left(1-n_{c h} \hat{\alpha}^{\prime}\right)
$$

We note that $\frac{d}{d t} y=\tilde{P}^{*}-\tilde{\beta}_{d} y$ also corresponds to the first order approximation of a $G C A P s^{-/}$photoreceptor with dark turnover rate $\tilde{\beta}_{d}$ and cyclase activity $\tilde{\alpha}=\tilde{\beta}_{d} c_{c g, d}$ (we assume 
that the dark cGMP concentration is unchanged). Because $\tilde{P}^{*}(t)$ is the same for WT and $\mathrm{GCAPs}^{-/}$photoreceptors, we thus find that as a first approximation that a WT photoreceptor is described by the equations for a $G C A P s^{-/-}$photoreceptor with increased dark turnover rate $\tilde{\beta}_{d}=\beta_{d}\left(1-n_{c h} \hat{\alpha}^{\prime}\right)$ and increased cyclase activity $\tilde{\alpha}=\tilde{\beta}_{d} c_{c g, d}$. This finding reveals a profound connection between the dim-flash responses of WT and $G C A P s^{-/-}$photoreceptors: the dim flash response (and also single-photon response) of a WT photoreceptor with dark turnover rate $\beta_{d}$ is identical to the dim-flash response of a $G C A P s^{-/-}$photoreceptor with increased dark turnover rate $\tilde{\beta}_{d}$ given by Eq. 7, thus confirming the importance of the dark GMP turnover rate for the kinetics of the flash response (Nikonov et al., 2000).

With the linear approximation $\beta_{d} H(y)=\tilde{\beta}_{d} y$, we obtain the solution

$$
y(t)=\kappa \xi \int_{0}^{t} \phi(s) g_{y}(t-s) d s
$$

with Green's function

$$
\begin{aligned}
g_{y}(t)= & \mu_{r h} \mu_{p d e} \mu_{t r}\left(-\frac{e^{-\mu_{r h} t}}{\left(\mu_{r h}-\mu_{t r}\right)\left(\mu_{r h}-\mu_{p d e}\right)\left(\mu_{r h}-\tilde{\beta}_{d}\right)}-\frac{e^{-\mu_{t r} t}}{\left(\mu_{t r}-\mu_{r h}\right)\left(\mu_{t r}-\mu_{p d e}\right)\left(\mu_{t r}-\tilde{\beta}_{d}\right)}\right. \\
& -\frac{e^{-\mu_{p d e} t}}{\left(\mu_{p d e}-\mu_{r h}\right)\left(\mu_{p d e}-\mu_{t r}\right)\left(\mu_{p d e}-\tilde{\beta}_{d}\right)}-\frac{\left.\tilde{\beta}_{d}-\mu_{r h}\right)\left(\tilde{\beta}_{d}-\mu_{t r}\right)\left(\tilde{\beta}_{d}-\mu_{p d e}\right)}{\left(\tilde{\beta}_{d}\right) .}
\end{aligned}
$$

Note that $\tilde{\beta}_{d} g_{y}(t)$ is symmetric in $\mu_{r h}, \mu_{p d e}, \mu_{t r}$ and $\tilde{\beta}_{d}$. For a short flash we have $y(t) \approx$ $R_{0}^{*} \xi g_{y}(t)$. The time course of the normalized cGMP concentration is $\hat{c}_{c g}(t)=e^{-\frac{y(t)}{n_{c h}}}=$ $e^{-\frac{R_{0}^{*} \xi}{n_{c h}} g_{y}(t)}$ 
If $\mu_{r h}$ and $\mu_{t r}$ are large compared to $\mu_{p d e}$ and $\tilde{\beta}_{d}$, the response at its maximum amplitude is governed by

$g_{y}(t) \approx \mu_{r h} \mu_{p d e} \mu_{t r}\left(-\frac{e^{-\mu_{p d e} t}}{\left(\mu_{p d e}-\mu_{r h}\right)\left(\mu_{p d e}-\mu_{t r}\right)\left(\mu_{p d e}-\tilde{\beta}_{d}\right)}-\frac{e^{-\widetilde{\beta}_{d} t}}{\left(\tilde{\beta}_{d}-\mu_{r h}\right)\left(\tilde{\beta}_{d}-\mu_{t r}\right)\left(\tilde{\beta}_{d}-\mu_{p d e}\right)}\right)$

From the condition for the derivative $g_{y}{ }^{\prime}\left(t_{\text {peak }}\right)=0$, we get for the time to peak

$$
t_{p e a k} \approx \frac{1}{\mu_{p d e}-\tilde{\beta}_{d}} \ln \left(\frac{\mu_{p d e}}{\tilde{\beta}_{d}} \frac{\left(\mu_{r h}-\tilde{\beta}_{d}\right)\left(\mu_{t r}-\tilde{\beta}_{d}\right)}{\left(\mu_{r h}-\mu_{p d e}\right)\left(\mu_{t r}-\mu_{p d e}\right)}\right)
$$

The peak amplitude $g_{y, p e a k}=g_{y}\left(t_{\text {peak }}\right)$ is

$$
g_{y, p e a k} \approx \frac{\mu_{p d e}}{\tilde{\beta}_{d}-\mu_{p d e}}\left(\left(\frac{\mu_{p d e}}{\tilde{\beta}_{d}}\right)^{\frac{\mu_{p d e}}{\widetilde{\beta}_{d}-\mu_{p d e}}}-\left(\frac{\tilde{\beta}_{d}}{\mu_{p d e}}\right)^{\frac{\widetilde{\beta}_{d}}{\mu_{p d e}-\widetilde{\beta}_{d}}}\right)
$$

Contrary to the time to peak, to compute the peak amplitude we can neglect the contributions from $\mu_{r h}$ and $\mu_{t r}$.

\section{Dynamics of saturating flash responses during the saturation phase}

For maximum-amplitude (saturating) responses with $y \gg 1$ and $\tilde{P}^{*} \gg 1$, we have $H(y) \approx$ $n_{c h} \hat{\alpha}(y) e^{\frac{y}{n_{c h}}}$ and $\frac{\frac{d}{d t} y}{\tilde{P}^{*}} \ll 1$. With maximal cyclase activation, $\alpha_{\max }=\hat{\alpha}_{\max } \beta_{d} c_{c g, d}$, we find from Eq. 1 that during saturation 


$$
y(t) \approx n_{c h} \ln \left(\frac{\tilde{P}^{*}(t)}{\hat{\alpha}_{\max } \beta_{d} n_{c h}}\right) .
$$

From this expression, we can obtain the GMP concentration as a function of the number of activated PDEs from:

$$
c_{c g}(t) \approx \frac{\alpha_{\max }}{\beta_{s u b} P^{*}(t)} .
$$




\section{RESULTS}

The signal transduction cascade of the vertebrate photoreceptor consists of an activation phase governed by excitation of rhodopsin, transducin, and cGMP phosphodiesterase-6 (PDE). Activated PDE increases the rate of hydrolysis of cGMP, which leads to a decline in the cGMP concentration, channel closure, and a reduction in $\mathrm{Na}^{+}$and $\mathrm{Ca}^{2+}$ current entering the outer segment. Current recovery depends upon the deactivation of all of the activation steps.

We recently introduced a novel method of analysis of the photoreceptor response, where we used equations for the $\log$ transform of the cGMP concentration, $y=-n_{c h} \ln \hat{c}_{c g}$ (Eq. 1), with the current given by $\hat{I}=e^{-y}$. We further introduce the more common current $\hat{\imath}=1-\hat{I}=1-$ $e^{-y}$ which is zero in darkness. In Fig. 1, we compare the dim flash responses of rods (Figs. 1A and 1B) and cones (Figs. 1C and 1D) with and without the GCAP proteins (black) to simulations of $y$ from Eqns. 1 - 3 (red), obtained with parameters from Table 2 which were derived in Reingruber et al. (2020), except for a slight modification in the values of the collecting areas to provide a better fit to the peak amplitudes. All the model parameters are defined in Table 1.

We now extend the analysis of our model and show (see Methods) that, for dim flashes, we can solve the model equations analytically to derive an expression for $y(t)$ for the entire current response, $y(t)=R_{0}^{*} \xi g_{y}(t)$, where $g_{y}(t)$ is given by Eq. 9 . The results of these calculations with parameters from Table 2 are shown as green curves in Fig. 1. In the following, we will use our analytic formulas to study in detail the kinetics of the dim flash responses in rods

and cones in order to reveal how underlying biophysical processes and parameters determine the waveform and amplitude of flash responses. This analysis will help us extract biophysical 
parameters from experimental recordings and provide a better understanding of the transduction cascade.

\section{Waveform of dim-flash responses}

The analytical results $y(t)=R_{0}^{*} \xi g_{y}(t)$ provide the important demonstration that not only the initial phase, but the entire dim-flash response is linear with the flash intensity $\phi$. We can then define the waveform of a dim-flash response as $\hat{y}(t)=\frac{y(t)}{y_{\text {peak }}}$, which is independent of the flash intensity. With the analytic result $\hat{g}_{y}(t)=\frac{g_{y}(t)}{g_{y, p e a k}}$, we have $y(t)=y_{\text {peak }} \hat{g}(t)$, where $y_{\text {peak }}=$ $R_{0}^{*} \xi g_{y, p e a k}$ is the amplitude of the response. Because the waveform is independent of the flash intensity, we can average over multiple waveforms to reduce experimental noise. In Fig. 2 we use the normalized rod and cone data from Fig. 1 (thin continuous lines) to compute the averaged waveform (thick continuous lines), which we compare with the analytic results $\hat{g}_{y}(t)$ computed with Eq. 9 and parameters from Table 2 (green lines). The waveform is much faster in a cone compared to a rod due to more rapid rates of response decay. The difference in the waveform between WT and $\mathrm{GCAPs}^{-/}$is the result of $\mathrm{Ca}^{2+}$ feedback to the cyclase, which changes the value of $\widetilde{\beta}_{d}$ defined in Eq. 7. The discrepancy between model and data, especially for rods, is more pronounced during the rising phase, where the computed waveform rises faster than the data. Because the response also rises faster in $\mathrm{GCAPs}^{-/-}$rods where $\mathrm{Ca}^{2+}$ feedback is absent, the discrepancy between model and data is unlikely to result from our assumptions about $\mathrm{Ca}^{2+}$. Instead, we think that the main reason for the discrepancy is our neglect of fast and intermediate processes in our model, for example multiple steps of phosphorylation of rhodopsin, which 
would further slow down the initial rise (see also Discussion and the blue curve in Fig. 3B for large $\left.\mu_{t r}\right)$.

This analysis shows that the waveform depends on $\hat{g}_{y}(\mathrm{t})$, which in turn depends only on the dynamical parameters $\mu_{r h}, \mu_{t r}, \mu_{p d e}$ and $\tilde{\beta}_{d}$ (see Methods, Eq. 9). We come to the surprising conclusion that the waveform of the photoreceptor response is determined exclusively by the deactivation rates $\mu_{r h}, \mu_{t r}$ and $\mu_{p d e}$, together with the effective cGMP turnover rate $\tilde{\beta}_{d}$, which additionally depends on $\mathrm{Ca}^{2+}$ feedback to the cyclase (see Eq. 7). In the following we simplify the notation and we generally refer to the parameters $\mu_{r h}, \mu_{t r}, \mu_{p d e}$ and $\tilde{\beta}_{d}$ as deactivation parameters or deactivation rates, although it is clear that $\tilde{\beta}_{d}$ is conceptually different from a deactivation rate like $\mu_{r h}$. The activation parameters $\beta_{s u b}$ and $k_{a c t}$ only scale the waveform by means of the gain $\xi$. We can therefore separate our analysis into two parts: (1), analysis of the rising and recovery phase of the waveform to extract information about the deactivation parameters; and (2), analysis of the peak amplitude $y_{\text {peak }}=R_{0}^{*} \xi g_{y, p e a k}$ to additionally infer information about the gain $\xi$ and rates of activation. Whereas the peak amplitude $y_{\text {peak }}$ depends on activation and deactivation parameters, the peak time is a property of the waveform and depends only on deactivation parameters (see Eq. 10).

\section{Rising phase of dim-flash responses}

Our analytic result for $g_{y}(t)$ in Eq. 9 describes the dim-flash response as a sum of exponentials. To examine whether a simpler polynomial approximation of the waveform exists during the rising phase, we have expanded $\hat{g}_{y}(t)=\frac{g_{y}(t)}{g_{y, p e a k}}$ for small values of the time $t$ in a Taylor series 
using Eq. 9. Note that the following analysis does not require us to have an explicit analytic expression for $g_{y, p e a k}$. The Taylor series expansion is alternating, $\hat{g}_{y}(t)=a t^{3}-b t^{4}+c t^{5}-$ $d t^{6}+\ldots$ with the leading-order coefficient $a=\frac{1}{6} \frac{\mu_{r h} \mu_{p d e} \mu_{t r}}{\mathrm{~g}_{y, p e a k}}$. Expressions for the higher order coefficients are lengthy, and we omit them; they can be computed with software like Maple or Mathematica.

In Fig. 3A we show the expansion for a WT rod; for $G C A P S^{-/-}$rods and for the cones, the analysis is similar. The Taylor expansion reveals that the leading order term is proportional to $t^{3}$, which reflects the three processes in Eq. 1 that contribute to PDE activation. In general, if $n$ firstorder processes contribute to PDE activation, the leading order asymptotic is $\sim t^{n}$ (Baylor et al., 1974). For example, by assuming that the intermediate step by which a $T^{*}$ is transformed into a $P^{*}$ is very fast, corresponding to the limit $\mu_{t r} \rightarrow \infty$, the leading-order asymptotic changes to the quadratic polynomial $\hat{g}_{y}(t)=\frac{1}{2} \frac{\mu_{r h} \mu_{p d e}}{\mathrm{~g}_{y, p e a k}} t^{2}$. Since the waveform at short times depends on the number of intermediate processes that contribute to PDE activation, the larger this number, the longer the delay in the initial rise of the response. Thus, an analysis of the short-time waveform gives information about the effective number of intermediate processes that contribute to the early phase of PDE activation. That $\hat{g}_{y}(t)$ for WT rod rises too fast in comparison to the data (Fig. 3B, red vs black curves) may be an indication that additional intermediate steps should be included (such as steps in rhodopsin phosphorylation) to delay the initial rise. We do not introduce such steps, because the kinetics and even number of phosphorylation steps required to extinguish $\mathrm{R}^{*}$ activity are presently unknown, and the purpose of this work is to provide a comprehensive analysis by focusing on basic transduction processes rather than to obtain the best possible fit. 
We next asked whether the leading-order term of the Taylor expansion can be used to fit the rising phase of the waveform up to larger times of the order the inflection time of the waveform. Fig. 3A and Fig. 3B for the limit $\mu_{t r} \rightarrow \infty$ show that the leading-order expression is a valid approximation only during a very short initial time period and fails at later times. We therefore conclude that the leading-order expansion is not suitable to describe the rising phase up to the inflection point. Nevertheless, many studies have shown that the quadratic approximation $y(t)=\frac{1}{2} R_{0}^{*} A\left(t-t_{e f f}\right)^{2}$ gives a good fit to the rising phase of the light response up to this point (Pugh \& Lamb, 1993). In this equation $t_{\text {eff }}$ is an effective time delay that accounts for fast intermediate processes, and $A$ is known as the amplification constant (Pugh \& Lamb, 1993). We also find that the rising phase of the waveform data can be well fit by a quadratic function of the form $\hat{y}(t)=\frac{1}{2} \hat{A}\left(t-t_{\text {eff }}\right)^{2}$ (Fig. 3B green curves). This same equation also gives a good fit to $\hat{g}_{y}(t)($ Fig. 3B), which confirms that model and data are consistent.

Why does our leading-order expression proportional to $t^{3}$ fail to fit the rising phase, whereas a lower order quadratic polynomial is well suited? The reason is that cancellations in the Taylor series due to the alternating summands produce an effective behavior of the response at later times that is very different from the leading order term. Whereas the leading-order behavior with many intermediate steps can be proportional to $t^{n}$, where $n$ can be large, the effective behavior at later times might well be approximated by a polynomial of much lower order, e.g. by a quadratic function. The difficulty is that we cannot derive this effective polynomial starting from Eq. 9, and the polynomial fit has therefore no clear theoretical basis and must be considered as only empirical. 
Because the amplification constant $\hat{A}$ was obtained by fitting of the waveform, its value can depend only on the deactivation parameters $\mu_{\mathrm{rh}}, \mu_{\mathrm{tr}}, \mu_{\mathrm{pde}}$, and $\tilde{\beta}_{d}$. Unfortunately, since we cannot mathematically derive the effective polynomial, we do not have an analytic formula for $\hat{A}$. It therefore remains unclear how $\hat{A}$ relates to the underlying deactivation parameters. Since the leading-order expansion with large $\mu_{t r}$ is also a quadratic polynomial, $\hat{g}_{y}(t)=\frac{1}{2} \frac{\mu_{r h} \mu_{p d e}}{\mathrm{~g}_{y, p e a k}} t^{2}$, we verified that this expression is not related to the quadratic fitting function (Fig. 3B dashed blue curve), and therefore it cannot be used to estimate $\hat{A}=\frac{\mu_{r h} \mu_{p d e}}{\mathrm{~g}_{y, p e a k}}$. We also note that the assumption that $\mu_{t r}$ is very large, corresponding to a model where $\mathrm{R}^{*}$ directly activates $\mathrm{P} *$ (Lamb \& Pugh, 1992; Pugh \& Lamb, 1993), strongly increases the discrepancy between data and model during the rising phase (Fig. 3B blue vs red curve).

The amplification constant $A$ defined by Lamb and Pugh is obtained by fitting the logtransform $\mathrm{y}(t)$ with the quadratic fitting function $\frac{1}{2} R_{0}^{*} A\left(t-t_{e f f}\right)^{2}$ (Pugh \& Lamb, 1993). In contrast, our amplification constant $\hat{A}$ is obtained by fitting the waveform $\hat{y}(t)$ with this fitting function. With $y(t)=y_{\text {peak }} \hat{y}(t) \approx y_{\text {peak }} \frac{1}{2} \hat{A}\left(t-t_{\text {eff }}\right)^{2}=\frac{1}{2} R_{0}^{*} \frac{y_{\text {peak }}}{R_{0}^{*}} \hat{A}\left(t-t_{\text {eff }}\right)^{2}$ we obtain the formula $A=\frac{y_{\text {peak }}}{R_{0}^{*}} \hat{A}$, which relates the value of $A$ to that of $\hat{A}$. With the $\operatorname{dim}$-flash expression $y_{\text {peak }}=R_{0}^{*} \xi g_{y, p e a k}$ we further get $A=\xi g_{y, p e a k} \hat{A}=n_{c h} k_{a c t} \beta_{\text {sub }} \frac{\hat{A} g_{y, p e a k}}{\mu_{r h} \mu_{p d e}}$. With the fitted values for $\hat{A}$ from Fig 3 , with $\xi$ from Table 2 , and with $g_{y, \text { peak }}=0.16$ extracted from a numerical solution of Eq. 1, we compute for rods that $A=\xi g_{y, p e a k} \hat{A} \approx 7 s^{-2}$ (fit of data) and $A \approx 9.7 s^{-2}$ (fit of $\hat{g}_{y}$ ), values that are within the wide range that can be found in the literature (Nikonov et al., 2006; Chen et al., 2010b; Majumder et al., 2013; Woodruff et al., 2014; Vinberg 
et al., 2015; Ingram et al., 2016). For a Gnat1 $1^{-/-}$cone we estimate $\hat{A} \approx 2047 s^{-2}$ by fitting the waveform data in Fig 2B. With $\xi$ from Table 2 and $g_{y, p e a k}=0.26$ (extracted from a numerical solution of Eq. 11), we compute $A=\xi g_{y, p e a k} \hat{A} \approx 0.95 s^{-2}$, less than for rods and similar to previous estimates (Nikonov et al., 2006; Ingram et al., 2019).

The value of $A$ can also be computed by extracting $\frac{y_{p e a k}}{R_{0}^{*}}$ from the data (see also Fig. 5), and by using this value together with the fitted value for $\hat{A}$ to compute $\mathrm{A}=\frac{y_{\text {peak }}}{R_{0}^{*}} \hat{A}$; or, alternatively, from the frequently used procedure of fitting individual dim-flash responses $y(t)$ (as in Pugh \& Lamb, 1993). All of these methods are equivalent and necessarily give similar results for $A$ (not shown) because, for dim flashes, $y(t)=y_{\text {peak }} \hat{y}(t)$ and $y_{\text {peak }}=R_{0}^{*} \xi g_{y, p e a k}$. It is however advantageous to use $\hat{A}$ for the computation of $A$, because the recording noise can be reduced by averaging over multiple waveforms of individual responses.

In a previous analysis, Pugh and Lamb (1993) derived the expression $A=n_{c h} k_{a c t} \beta_{\text {sub }}$ and inferred that $A$ depends only on activation parameters. To derive their formula, they considered that the intermediate steps of transducin activation proceed very rapidly, corresponding to the limit $\mu_{t r} \rightarrow \infty$, and they further neglected all deactivation processes, corresponding to the limits $\mu_{r h} \rightarrow 0, \mu_{p d e} \rightarrow 0$ and $\tilde{\beta}_{d} \rightarrow 0$. With these approximations, the rise of the waveform is given by the first order term $\hat{g}_{y}(t)=\frac{1}{2} \frac{\mu_{r h} \mu_{p d e}}{\mathrm{~g}_{y, p e a k}} t^{2}$ (blue dashed curve in Fig 3B) such that $\hat{A}=\frac{\mu_{r h} \mu_{p d e}}{\mathrm{~g}_{y, \text { peak }}}$. The corresponding expression for the log-normal response is $\mathrm{y}(t)=$ $R_{0}^{*} \xi g_{y, p e a k} \hat{g}_{y}(t)=\frac{1}{2} R_{0}^{*} n_{c h} k_{a c t} \beta_{\text {sub }} t^{2}$. Hence, in the limit $\mu_{t r} \rightarrow \infty$ with vanishing deactivation rates, the rise of the response is described by a quadratic function with amplification constant $A=n_{c h} k_{a c t} \beta_{\text {sub }}$, just as Pugh and Lamb obtained. This result for $A$ is a special case of 
our general formula $A=n_{c h} k_{a c t} \beta_{\text {sub }} \frac{\hat{A} g_{y, p e a k}}{\mu_{r h} \mu_{p d e}}$ for $\frac{\hat{A} g_{y, p e a k}}{\mu_{r h} \mu_{p d e}}=1$, which results from the first order expression $\hat{A}=\frac{\mu_{r h} \mu_{p d e}}{\mathrm{~g}_{\text {y,peak }}}$.

The amplification constant $A=n_{c h} k_{a c t} \beta_{s u b}$ faithfully characterizes the response for the case $\mu_{t r} \rightarrow \infty$ but only under two conditions. First, the deactivation rates must all be zero, in which case the response rises indefinitely as $\mathrm{y}(t)=\frac{1}{2} R_{0}^{*} n_{c h} k_{a c t} \beta_{s u b} t^{2}$. The value $A=$ $n_{c h} k_{a c t} \beta_{\text {sub }}$ therefore corresponds to the maximal amplification that this system would achieve if all deactivation processes are switched off. In this case, $A$ necessarily depends only on activation rates. Second, during some very early phase of the response where the waveform can be approximated by the first order term of its Taylor expansion (Fig. 3A and 3B), the response rises with maximal amplification. The exact extent of this early phase depends on the deactivation rates. It is certainly not appropriate to fit the data up to the inflection point of the rising phase without considering deactivation processes, since the inflection point is a clear manifestation of the impact of the deactivation rates: without deactivation, this point would not exist and the response would rise indefinitely. If the data are fitted up to the inflection point, deactivation rates will necessarily affect the value of the amplification constant, which is quantified by our formula $\frac{\hat{A} g_{y, p e a k}}{\mu_{r h} \mu_{p d e}}$.

Our formula $A=n_{c h} k_{a c t} \beta_{s u b} \frac{\hat{A} g_{y, p e a k}}{\mu_{r h} \mu_{p d e}}$ is valid for general $\mu_{t r}$, and the latter affects the value of $\hat{A} g_{y, p e a k}$. By further considering the possibility that $\mathrm{T}^{*}$ deactivation can occur also without activation of $\mathrm{P}^{*}$, in which case the values of $k_{t r}$ and $\mu_{t r}$ would be different, our formula would generalize to $A=n_{c h} k_{a c t} \beta_{s u b} k_{t r} \frac{\hat{A} g_{y, p e a k}}{\mu_{r h} \mu_{p d e} \mu_{t r}}$. 
In summary, we conclude that fitting the rising phase of the waveform with a quadratic expression is empirical but provides no precise information about the underlying parameters because we do not have an analytic expression for $\hat{A}$. To estimate precise values for the deactivation rates from a waveform analysis, we must use Eq. 9. We return to the relationship between amplification and activation/inactivation rates in the Discussion.

\section{Recovery phase of dim-flash responses}

Both the rising and recovery phase of the waveform are governed by the same sum of exponentials given in Eq. 9. During the initial rising phase when time is small, all exponentials contribute to the waveform. Around peak time, the contributions from at least two exponentials are relevant, because a single exponential cannot generate a peak in the waveform. Finally, at larger times during the recovery phase, only the exponentials with the smallest rate constants (and slowest decay) contribute significantly to the waveform. For the values of parameters from

Table 2, we have that $\mu_{r h}$ and $\mu_{t r}$ are much larger than $\mu_{p d e}$ and $\tilde{\beta}_{d}$, where we can assume $\tilde{\beta}_{d}=$ $\beta_{d}$ for $G C A P s^{-/}$and $\tilde{\beta}_{d} \approx 3.8 \beta_{d}$ for WT photoreceptors (see Eq. 7). We conclude that the recovery phase of dim-flash responses in both rods and cones is governed primarily by the decay of light-activated $\mu_{p d e}$ and the rate $\tilde{\beta}_{d}$ (note that $\tilde{\beta}_{d}$ depends on the dark turnover rate and degree of the cyclase activation). Moreover, from Table 2 we can compute for a WT rod that $\tilde{\beta}_{d} \approx$ $15.6 s^{-1}$, and since this value is much larger than $\mu_{p d e}=5 s^{-1}$, it follows that the recovery of WT rod flash response is well approximated by a single exponential with decay rate $\mu_{p d e}$, as previous experiments have indicated (Krispel et al., 2006; Tsang et al., 2006; Chen et al., 
2010a). Similarly, for a $G C A P s^{-/}$cone with $\beta_{d}=11 s^{-1}$ and $\mu_{p d e}=37.8 s^{-1}$, we conclude that the recovery is governed by a single exponential with rate $\beta_{d}$.

To verify these conclusions, we fitted the recovery phase of the averaged waveform derived from the WT rod and the Gnat $1^{-\varkappa} ;$ GAPS $^{-/}$cone from Fig 1 (black), with a single exponential (red) to estimate the decay rate $\mu_{r e c}$ (Fig. 4A-B). From the fitting of the averaged waveform, we obtained $\mu_{\text {rec }}=4.9 \mathrm{~s}^{-1}$ for the WT rod and $\mu_{\text {rec }}=11.9^{-1}$ for the Gnat $^{--} ; G C A P s^{-/-}$cone, which are both similar to the values in Table 2 that were obtained by fitting entire responses in Reingruber et al. (2020). By fitting the recovery of the three individual traces that were used to generate the averaged waveform, we get $\mu_{r e c}=4.9 \pm 0.8 \mathrm{~s}^{-1}$ and $\mu_{\text {rec }}=12.4 \pm 1.2 \mathrm{~s}^{-1}$ (mean \pm S.E.). In contrast, for a $G C A P \mathrm{~s}^{-/}$rod the parameters $\beta_{d}=4.1 \mathrm{~s}^{-1}$ and $\mu_{p d e}=5 s^{-1}$ have very similar values, with the consequence that the recovery depends upon a sum of two exponentials. That is also true for a WT cone where $\tilde{\beta}_{d} \approx 41.8 s^{-1}$ is similar to $\mu_{p d e}=37.8 s^{-1}$.

To illustrate these findings, we compare the recovery between a WT and $G C A P s^{-/-}$rod (Fig. 4C-D). We use Eq. 9 to extract the different exponential contributions to the waveform related to rhodopsin decay, transducin decay, PDE decay and the cyclase, $\hat{g}_{y}=\hat{g}_{y, r h}+\hat{g}_{y, t r}+$ $\hat{g}_{y, p d e}+\hat{g}_{y, \beta}$ (Fig. 4C-D). Whereas the recovery of a WT rod (Fig. 4C, black curve) is dominated by a single exponential related to PDE decay (Fig. 4C, red curve), the recovery of a GCAPs $^{-/-}$rod (Fig. 4D, black curve) is the sum of two exponentials with decay rates $\mu_{p d e}$ (Fig. $4 \mathrm{D}$, red curve) and $\beta_{d}$ (Fig. 4D, blue curve). 


\section{Peak amplitude, gain and sensitivity of dim-flash responses}

The waveform analysis provides information about the dynamical parameters $\mu_{r h}, \mu_{t r}, \mu_{p d e}$, and $\tilde{\beta}_{d}$. The activation parameters $\beta_{\text {sub }}$ and $k_{a c t}$ can be extracted from the gain $\xi$. Since $y_{p e a k}=$ $R_{0}^{*} \xi g_{y, p e a k}$, we can learn about $\xi$ from the peak amplitude of the responses. The values $y_{\text {peak }}$ can be extracted from the data, and if the collecting area $\kappa$ is known, the expected number of activated rhodopsin molecules can be computed as $R_{0}^{*}=\kappa \phi \Delta t$. To complete this calculation, however, we need to estimate $g_{y, p e a k}$.

Because we cannot extract $g_{y, p e a k}$ directly from the data, we need additional information about the dynamical parameters in order to compute $g_{y, p e a k}$ either from Eq. 9 or Eq. 11, or from a simulation obtained with Eq. 1. If the waveform around peak time is governed by $\mu_{p d e}$ and $\tilde{\beta}_{d}$, we can use Eq. 11 to compute $g_{y, p e a k}$ as a function of $\frac{\mu_{p d e}}{\widetilde{\beta}_{d}}$. Because the value of $g_{y, p e a k}$ changes only slowly as a function of the dynamical parameters, acceptable values for $g_{y, p e a k}$ can be computed even if the dynamical parameters are not known precisely. With Eq. 11 and parameters from Table 2, we obtain $g_{y, p e a k}=0.19(\operatorname{rod} \mathrm{WT}), g_{y, p e a k}=0.40\left(\operatorname{rod} G C A P s^{-/}\right)$, $g_{y, \text { peak }}=0.34\left(\right.$ cone Gnat $\left.^{-/-}\right), g_{y, \text { peak }}=0.60\left(\right.$ cone Gnat $^{-/-} ;$GCAPs $\left.^{-/-}\right)$. For comparison, from a numerical simulation of Eq. 1, we find $g_{y, p e a k}=0.16(\operatorname{rod} \mathrm{WT}), g_{y, p e a k}=0.39(\operatorname{rod}$ $\left.G C_{A P s^{-/}}\right), g_{y, \text { peak }}=0.26\left(\right.$ cone Gnat1 $\left.^{-/}\right), g_{y, \text { peak }}=0.55\left(\right.$ cone Gnat1 $^{-/-} ;$GCAPs $\left.^{-/-}\right)$. Because the values of $g_{y, p e a k}$ are similar between rods and cones, the large difference in the response amplitude between rods and cones is primarily generated by the difference in the gain $\xi$, which is of the order $0.45 / 0.0018 \approx 250$ (see Table 2). 
In Fig. 5, we plot $\frac{y_{\text {peak }}}{g_{y, \text { peak }}}$ against $R_{0}^{*}$ for rods and cones, with $g_{y, p e a k}$ computed from Eq. 1 and the parameters in Table 2. Because $\xi$ is the same for WT and GCAPs $s^{-/}$photoreceptors, we can combine their data to estimate $\xi$. For a rod we obtain $\xi=0.42$ (Fig. 5A), and for cone we get $\xi=0.0019$ (Fig. 5B), which are both close to the values in Table 2 obtained by collective fitting of all of the responses. The gain is closely connected to the flash sensitivity. If we define the flash sensitivity $S_{f}$ of a dark-adapted photoreceptor as the derivative of the normalized peakresponse amplitude with respect to the number of photoisomerizations $R_{0}^{*}$, then for dim flashes with $\hat{\imath}_{\text {peak }} \approx y_{\text {peak }}$ we have $S_{f} \approx \xi g_{y, p e a k}$. With the approximation $g_{y, p e a k, \text { rod }} \approx g_{y, p e a k, \text { cone }}$, the sensitivity ratio between rods and cones is given simply the ratio of the gain, $\frac{S_{f, \text { rod }}}{S_{f, \text { cone }}} \approx \frac{\xi_{\text {rod }}}{\xi_{\text {cone }}}$. Hence to first estimation, the gain difference between rods and cones can be obtained from the ratio of the flash sensitivities, which can be extracted from the data. This conclusion is more important than it may seem, because it implies that the sensitivity of the photoreceptor is

determined by the ratio $\frac{n_{c h} \beta_{s u b} k_{a c t}}{\mu_{p d e} \mu_{r h}}$. Assuming that the channel cooperativity $n_{c h}$ is the same for rods and cones, the difference in sensitivity of the two kinds of photoreceptors can then be seen to depend only on the properties of the three proteins rhodopsin, transducin and PDE, which are all present as different isoforms in rods and cones (see Ingram et al., 2016).

\section{Dynamics of saturating flash responses}

So far, we have focused on dim-flash responses where we made use of our analytic result in Eq.

9. We do not use our model to fit brighter-light and saturating flash responses, because the model does not incorporate adaptation processes. It is nevertheless insightful to discover what 
the model predicts for saturating flashes. To study the responses to bright flashes we start from Eq. 1. Since for saturating flashes the normalized current $\hat{\imath}$ is close to one and almost constant during the saturation phase, the current does not reveal much information about the dynamics of PDE activity and cGMP concentration during this phase. We therefore focus on the dynamics of the log-transform $y(\mathrm{t})$.

In Fig. 6 we compare the time course of $y(t)$ computed from Eq. 1 for saturating-flash responses for WT and GCAPs ${ }^{-/-}$rods (Fig. 6A), and Gnat1 ${ }^{-/-}$(effectively WT) and Gnat1 $^{-/} ; \mathrm{GCAPs}^{-/-}$cones (Fig. 6B). Whereas for dim flashes the responses are different between WT and $\mathrm{GCAPs}^{-/-}$photoreceptors due to the cyclase, the kinetics during the saturation period become similar because the cyclase is maximally activated and constant and therefore does not affect the time dependency of the response. From Eq. 13, we find that the cGMP dynamics during saturation are determined by the PDE dynamics such that the product of the cGMP concentration and the number of activated PDEs is approximately constant with time. The magnitude of cyclase activation modulates the level of cGMP synthesis and thereby affects the extent by which the cGMP concentration is depleted by activated PDE (cGMP becomes less depleted in a WT photoreceptor due to higher cyclase activity). The level of cyclase activation affects the maximum of $y$ that is attained during saturation. However, the shape of the responses, which can be found by normalizing the response to the maximum, is governed by PDE dynamics which are the same in both photoreceptors. This is the reason why in Figs. 6A and 6B we can adjust the flash intensity to superimpose responses for WT and $G C A P s^{-/}$photoreceptors during the saturation phase.

Eq. 13 predicts that during the saturation phase, the cGMP concentration adapts to PDE activation such that the hydrolysis rate matches the synthesis rate, $\beta_{s u b} P^{*}(t) c_{c g}(t) \approx \alpha_{\max }$. 
More generally, the ratio of the synthesis to hydrolysis rate during a flash response evolves according to

$$
r=\frac{\alpha}{\beta_{d} c_{c g}+\beta_{s u b} P^{*} c_{c g}}=\frac{\hat{\alpha}}{\hat{\alpha}+\frac{\hat{c}_{c g}}{n_{c h} \beta_{d}} \frac{d y}{d t}}=\frac{\hat{\alpha}}{\hat{\alpha}-\frac{1}{\beta_{d}} \frac{d \hat{c}_{c g}}{d t}} .
$$

At peak time we have $\frac{d y}{d t}=0$ and $r=1$. In Figs. 6C and 6D, we show $r$ from Eq. 14 for the two strongest flashes from Figs. 6A and 6B. At the beginning, $r$ quickly decreases due an increase in cGMP hydrolysis rate driven by PDE activation. This decrease in $r$ produces rapid cGMP depletion, which in turn decreases the hydrolysis rate so that $r$ starts to increase again even for $\mathrm{GCAPs}^{-/}$photoreceptors in the absence of cyclase feedback. At the time-to-peak of the response, the synthesis and hydrolysis of cGMP are the same, and $r=1$. The value of $r$ stays close to one during the saturation phase (Figs. 6C and 6D), which can be inferred from Eq. 14 because $\left|\frac{\hat{c}_{c g}}{n_{c h} \beta_{d}} \frac{d y}{d t}\right| \ll 1$ when $\hat{c}_{c g}$ is small. Finally, during the recovery phase, $r$ increases because the cyclase rate is greater than the PDE rate, and $r$ returns to its equilibrium value as the response decays to baseline.

Figs. 6A and 6B show that during saturation, $y(t)$ decreases almost linearly as a function of time. To explain this quasi-linearity, we assume that PDE recovery is governed by $\mu_{p d e}$ such that $g_{p}(t) \approx \eta e^{-\mu_{p d e} t}\left(\right.$ Eq. 5). With $\widetilde{P}^{*}(t) \approx R_{0}^{*} \xi \eta e^{-\mu_{p d e} t}=\widetilde{P}_{\text {max }}^{*} e^{-\mu_{p d e} t}$ we obtain from Eq. 12 during saturation

$$
\frac{y(t)}{n_{c h}} \approx \ln \left(\frac{\tilde{P}_{\max }^{*}}{\hat{\alpha}_{\max } \beta_{d} n_{c h}}\right)-\mu_{p d e} t=\ln \left(\frac{R_{0}^{*} \xi \eta}{\hat{\alpha}_{\max } \beta_{d} n_{c h}}\right)-\mu_{p d e} t .
$$


Eq. 15 reveals that $y(t) / n_{c h}$ decreases linearly with time with a slope given by $\mu_{p d e}$. Unfortunately, it is difficult to exploit Eq. 15 to estimate $\mu_{p d e}$ by fitting the decay of $y(t)$, because we cannot reliably compute $y(t)$ from the data when the current is close to 1 as a result of noise and the singularity of the logarithm at zero. Instead, to extract $\mu_{p d e}$ we can use information from multiple flash responses to measure the time $t_{D}$ when the recovering current crosses a fixed threshold $\hat{\imath}_{D}$, corresponding to the fixed value $y_{D}=-\ln \left(1-\hat{\imath}_{D}\right)$. With Eq. 15 we find that $t_{D}$ satisfies $-\frac{y_{D}}{n_{c h}}+\ln \left(\frac{\xi \eta}{\widehat{\alpha}_{\max } \beta_{d} n_{c h}}\right)+\ln R_{0}^{*}-\mu_{p d e} t_{D} \approx 0$. Since the first two terms in this expression are constants, one can extract $\mu_{p d e}$ by fitting the slope of $t_{D}$ as a function of $\ln R_{0}^{*}$, which is called a Pepperberg plot after the late David Pepperberg (Pepperberg et al., 1992). This analysis shows that the dominant time constant in this model is given by the PDE deactivation rate, $\tau_{D}=\mu_{p d e}{ }^{-1}$, and that a Pepperberg analysis can in principle be performed with both WT and $G C A P s^{-/}$responses. 


\section{DISCUSSION}

The signal transduction pathway of the vertebrate rod and cone photoreceptor consists of a series of biochemical and biophysical processes that transform the absorption of a photon into a change in membrane current. Despite many sophisticated mathematical models, a precise conceptual understanding of how all these interconnected processes work together to generate the response remains challenging. To obtain such an understanding, we derived an analytic solution for the entire dim-flash response based on a representative model that comprises the most important transduction processes. We applied this solution to dissect the flash response and to investigate its characteristic phases: the activation phase where the current rises, the intermediate peak or saturation phase, and the recovery phase where the photoreceptor returns to its initial state. Our novel analysis provides a detailed characterization of these phases.

Our analytic result $y(t)=R_{0}^{*} \xi g_{y}(t)$ shows that the entire log-transform of the dim-flash response is linear with the number of photoisomerizations $R_{0}^{*}$ generated by the flash. Since for $\operatorname{dim}$ flashes we have $\hat{\imath}(\mathrm{t})=1-e^{-\mathrm{y}(\mathrm{t})} \approx \mathrm{y}(\mathrm{t})$, to a first approximation the current is also linear with $R_{0}^{*}$. The analytic function $g_{y}(t)$ (Eq. 9) depends entirely on the deactivation parameters $\mu_{r h}, \mu_{t r}, \mu_{p d e}$ and $\tilde{\beta}_{d}$, where $\tilde{\beta}_{d}$ can be seen as an effective dark cGMP turnover rate that also depends on $\mathrm{Ca}^{2+}$-feedback to the cyclase (Eq. 7). The activation parameters $\beta_{\text {sub }}$ and $k_{\text {act }}$ affect the response by means of the gain $\xi=\frac{n_{c h} k_{a c t} \beta_{s u b}}{\mu_{r h} \mu_{p d e}}$ (Eq. 2). We used this analytic result to separate the response into the waveform $\hat{y}(t)=\mathrm{g}_{y}(t) / g_{y, p e a k}$, which is independent of the flash intensity and is normalized to amplitude one (Fig. 2); and the amplitude $y_{\text {peak }}=R_{0}^{*} \xi g_{y, p e a k}$, such that $y(t)=y_{\text {peak }} \hat{y}(t)$. It follows that the dim-flash waveform $\hat{y}(t)$ depends entirely on the 
deactivation parameters $\mu_{r h}, \mu_{t r}, \mu_{p d e}$ and $\tilde{\beta}_{d}$, whereas the activation parameters $\beta_{s u b}$ and $k_{a c t}$ only affect the response amplitude $y_{\text {peak }}$.

In a previous attempt to analyze the rising phase of dim flashes, Pugh and Lamb (1993) found that the quadratic expression $y(t)=\frac{1}{2} R_{0}^{*} A\left(t-t_{\text {eff }}\right)^{2}$ gives a reasonable fit to the rising phase of the log-transform of the current. They called the parameter $A$ the amplification constant. By neglecting deactivation processes, Pugh and Lamb derived the formula $A=n_{c h} \beta_{\text {sub }} k_{a c t}$. This formula relates the fitted value of $A$ entirely to the activation rates and the channel cooperativity. This equation for $A$ has since been widely used to evaluate the initial activation process and to extract values for the rates $\beta_{\text {sub }}$ and $k_{a c t}$ (see for example Pugh \& Lamb, 2000; Kawamura \& Tachibanaki, 2008; Invergo et al., 2014; Astakhova et al., 2015; Lamb et al., 2018).

From our analysis of the rising phase, we have derived the new formula $A=\frac{y_{p e a k}}{R_{0}^{*}} \hat{A}$, where the amplification constant $\hat{A}$ can be obtained by fitting the rising phase of the waveform $\hat{y}(t)$ with the quadratic fitting function $\frac{1}{2} \hat{A}\left(t-t_{\text {eff }}\right)^{2}$ (Fig 3B). With our analytic result $y_{\text {peak }}=$ $R_{0}^{*} \xi g_{y, p e a k}$, we obtain $A=\xi g_{y, p e a k} \hat{A}=n_{c h} k_{a c t} \beta_{\text {sub }} \frac{\hat{A} g_{y, p e a k}}{\mu_{r h} \mu_{p d e}}$, where $\frac{\hat{A} g_{y, p e a k}}{\mu_{r h} \mu_{p d e}}$ depends only on the deactivation parameters. Our analysis thus reveals that the amplification constant $A$ of Pugh and Lamb depends on both activation and deactivation parameters, and the impact of the deactivation parameters is quantified by $\frac{\hat{A} g_{y, p e a k}}{\mu_{r h} \mu_{p d e}}$. For example, for a WT mouse rod with the fitted value $\hat{A}=97 \mathrm{~s}^{-2}$ (Fig 3B), $g_{y, p e a k}=0.16$ (from a numerical solution of Eq. 11), $\mu_{r h}=$

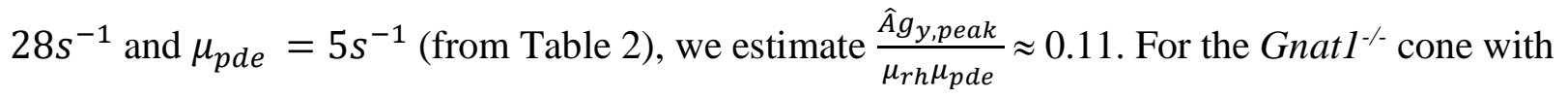


$\hat{A}=2047 \mathrm{~s}^{-2}$ (by fitting the data in Fig. 2B, not shown) and $g_{y, p e a k}=0.26$, we find $\frac{\hat{A} g_{y, p e a k}}{\mu_{r h} \mu_{p d e}} \approx$

0.2 . Hence, by assuming that $n_{c h} \approx 2.5$, we conclude that the incorrect formula $A=n_{c h} k_{a c t} \beta_{\text {sub }}$ would underestimate $k_{\text {act }} \beta_{\text {sub }}$ in a mouse rod by a factor of around 9 , and in a mouse cone by a factor of around 5. A similar underestimation by factors of around 4 and 1.6 have been inferred for a carp cone and a frog rod from simulations where the deactivation processes have been switched off (Astakhova et al., 2015).

The results of Pugh and Lamb (1993) were derived from a first-order analysis of model equations, which is unaffected by deactivation processes. As suggested by the frog result from (Astakhova et al., 2015), deactivation processes might have only a reduced impact in amphibian rods, which were used for the initial analysis of Pugh and Lamb (1993). Nevertheless, based on the observation that the rising phase of many rod and cone photoreceptors can be well approximated by the quadratic fitting function $y(t)=\frac{1}{2} R_{0}^{*} A\left(t-t_{\text {eff }}\right)^{2}$, Pugh and Lamb generalized their result without performing a mathematical validation (Pugh \& Lamb, 1993, 2000).

We derived an analytic solution for the dim-flash response that provided the basis for our analysis, and which revealed that the contribution of deactivation processes to the rising phase cannot be neglected. Moreover, we find that the fitting function $y(t)=\frac{1}{2} R_{0}^{*} A\left(t-t_{\text {eff }}\right)^{2}$ has to be considered as empirical because it is not derived from an analysis of the underlying model equations. Although our formula $A=\frac{y_{\text {peak }}}{R_{0}^{*}} \hat{A}$ shows that the amplification constant defined by Pugh and Lamb depends on activation and deactivation parameters, we do not have an analytic expression for $\hat{A}$. It is therefore unclear how to extract deactivation rates from the fitted value of 
$\hat{A}$. To estimate such values, one has to use Eq. 9 for a fitting procedure. Alternatively, in more complex models where no such analytic result is available, deactivation rates have to be estimated from the underlying model equations with numerical fitting procedures (see e.g. Hamer et al., 2005; Invergo et al., 2014).

The formula $A=n_{c h} k_{a c t} \beta_{\text {sub }}$ predicts that $\mathrm{Ca}^{2+}$-feedback does not affect the rising phase of dim-flash responses, which seems to be consistent with experimental data showing that the initial rise of the response is not much different between WT and $G C A P^{-/}$photoreceptors (Burns et al., 2002). In contrast, in our model $\mathrm{Ca}^{2+}$-feedback to the cyclase affects the rising phase of the waveform via the effective parameter $\tilde{\beta}_{d}$ (Eq. 7), and also affects the fitted value of the amplification constant $\hat{A}$. Since the time to peak is larger in a $G C A P s^{-/}$photoreceptors, the amplification constant $\hat{A}$ is necessarily smaller in a $G C A P^{-/}$photoreceptor. For example, by fitting the rod GCAPs ${ }^{-1}$ data from Fig. $2 \mathrm{~A}$, we find $\hat{A} \approx 42 \mathrm{~s}^{-2}$ (not shown), contrary to $\hat{A} \approx 97 \mathrm{~s}^{-2}$ for a WT rod (Fig. 3). Since however the response amplitude $y_{\text {peak }}$ is larger in a $G C A P s^{-/}$photoreceptor, the value of the amplification constant $A=\frac{y_{p e a k}}{R_{0}^{*}} \hat{A}=$ $n_{c h} k_{a c t} \beta_{\text {sub }} \frac{\hat{A} g_{y, p e a k}}{\mu_{r h} \mu_{p d e}}$ remains largely unchanged between WT and $G C A P s^{-/-}$photoreceptors. For example, for a WT rod we have $\hat{A} g_{y, p e a k} \approx 15.5 s^{-2}$, and for a $\mathrm{GCAPs}^{-/-} \operatorname{rod} \hat{A} g_{y, p e a k} \approx$ $16.4 s^{-2}$. We thus find that the experimental result that the initial rise of the response is not much different between WT and $G C A P^{-/}$photoreceptors does not imply that $\mathrm{Ca}^{2+}$-feedback does not affect the rising phase of dim-flash responses. We show that the initial rise of the response remains almost unchanged because $\mathrm{Ca}^{2+}$-feedback affects both response amplitude and response dynamics. Lamb and Pugh derived their formula $A=n_{c h} k_{a c t} \beta_{\text {sub }}$ by arguing that the $\mathrm{Ca}^{2+}$ dynamics is slow and therefore does not affect the initial rising phase. In contrast, we assume 
that $\mathrm{Ca}^{2+}$-dynamics is determined by the current dynamics, in which case $\mathrm{Ca}^{2+}$ feedback gradually modulates the response. By performing simulations with an additional equation for the $\mathrm{Ca}^{2+}$ concentration, we checked that our assumption is justified to model the rising phase of dimflash responses where the rise of the current is sufficiently slow. For strong flashes, where the current rises very fast, the initial $\mathrm{Ca}^{2+}$ dynamics is slightly delayed with respect to the current.

Because activation rates affect the gain $\xi$ (Eq. 2), analysis of the gain is a more effective way to extract information about activation parameters. We show that the gain can be estimated either from an analysis of the rising phase with the formula $\xi=\mathrm{A} /\left(\hat{A} g_{y, p e a k}\right)$, or by fitting entire flash responses as shown in Reingruber et al. (2020), or from an analysis of the response amplitude (Fig. 5). For example, for a WT rod, with the gain and deactivation parameters from Table 2 together with the assumption $\beta_{\text {sub }} \sim 0.02 s^{-1}$, we find $k_{\text {act }}=\xi \frac{\mu_{r h} \mu_{p d e}}{n_{c h} \beta_{s u b}} \approx 1260 s^{-1}$. With the amplification constant $\mathrm{A}=7.1 \mathrm{~s}^{-2}$ (estimated from Fig 3) together with the formula $A=$ $n_{c h} k_{a c t} \beta_{\text {sub }} \frac{\hat{A} g_{y, p e a k}}{\mu_{r h} \mu_{p d e}}$, we obtain $k_{a c t}=\frac{\mathrm{A}}{n_{c h} \beta_{\text {sub }}} \frac{1}{0.11} \approx 1290 \mathrm{~s}^{-1}$, which is consistent with the value estimated from the gain. In contrast, the Pugh and Lamb formula predicts a value $k_{a c t}=$ $\frac{A}{n_{c h} \beta_{s u b}} \approx 142 s^{-1}$. Because our estimation of the transducin activation rate is similar to the values of Heck and Hofmann (2001) and Lamb and Kraft (2020), and because we have used a conventional model of single-transducin activation for PDE, our calculations indicate that a high transducin activation rate cannot be used as unambiguous evidence for a dimeric transducin activation of PDE (Lamb \& Kraft, 2020).

The recovery phase of the current is frequently fitted with a single exponential to extract the recovery rate constant $\mu_{\text {rec }}$ (or its inverse $\tau_{\text {rec }}$ ) In WT rods this value is usually identified 
with the PDE deactivation rate $\mu_{p d e}$ (Krispel et al., 2006; Tsang et al., 2006; Chen et al., 2010a). We have shown, however, that a more accurate procedure is to fit the recovery of the logtransform of the current, which is more directly governed by exponential decays (Eq. 9). Moreover, because the recovery of the response depends on a sum of exponentials (Eq. 9), careful analysis is needed to interpret the fitted value for the recovery rate correctly. We show that fitting the recovery phase with a single exponential is justified only for WT rod and GCAPs $^{-/}$cone responses, where the fitted recovery rates correspond to $\mu_{p d e}$ (rod) and $\beta_{d}$ (cone). In contrast, for WT cone and GCAPs ${ }^{-/}$rod responses (Fig. 4C-D), the recovery phase is governed by a sum of two exponentials, and in this circumstance it is not appropriate to extract parameters by fitting the recovery phase with a single exponential decay function. For example, when Gross et al. (2012b) determined the widely accepted value $\beta_{d}=4.1 \mathrm{~s}^{-1}$, they claimed that PDE decay does not affect the recovery of $G C A P s^{-/}$rod responses. Although the latter is not correct, their estimation for $\beta_{d}$ seems still to be justified because it was apparently derived from $G C A P s^{-1-R G S 9-o x}$ rod responses with overexpressed GAP proteins, where PDE decay is accelerated to a value $\mu_{p d e} \approx 12.5 s^{-1}$.

Finally, we used the log-transform of the current to analyze response dynamics during saturation. Whereas the current reaches a maximal value and is almost constant during the saturation period, the log-transform of the current is not constant and can be used to study dynamics during saturation (Fig. 6). Although our model does not include mechanisms of adaptation and cannot be used to fit responses to brighter light intensities, the model predictions for saturating flashes are nevertheless interesting because they provide insight into the dynamics during the saturation period when cyclase is maximally activated. 
We find that during saturation, response dynamics are determined by a close interplay between PDE activation and cGMP depletion, such that the product of GMP concentration and the number of activated PDEs is approximately constant with time (Eq. 13). The extent of cyclase activation modulates the minimal level of the cGMP concentration that is attained, as a result of hydrolysis by activated PDE; but cyclase activity does not otherwise affect the dynamics of the response. This finding might have important implications for adaptation, because it suggests that modulation of PDE deactivation is a powerful means to control the cGMP concentration at high light intensities when the cyclase rate is a maximal value.

Our results for saturating flashes further provide a mathematical explanation for Pepperberg plots (Pepperberg et al., 1992). Eq. 15 predicts that the dominant time constant for a WT rod will be determined by the PDE deactivation rate, $\tau_{D}=\mu_{p d e}{ }^{-1}$, which agrees with experimental observations (Krispel et al., 2006; Tsang et al., 2006; Chen et al., 2010a). If PDE activation were super-linear due to the necessity of binding of two transducins such that $\tilde{P}_{\text {max }}^{*} \sim R_{0}^{*} e^{\rho R_{0}^{*}}$ (Lamb \& Kraft, 2020), Eq. 15 predicts $\tau_{D}=\frac{1+\rho R_{0}^{*}}{\mu_{p d e}}$, which agrees with the formula from Lamb and Kraft (2020). However, more precise analysis of a model that additionally incorporates adaptation processes is needed to clarify whether the observed increases of the dominant time constant with brighter flash intensity are a sign of dimeric PDE activation (Lamb \& Kraft, 2020), or of some other process (Martemyanov et al., 2008; Burns \& Pugh, 2010).

Whereas our model faithfully reproduces cone data, the discrepancy between model and data is larger for rods, especially during the initial phase where the simulations rise faster than the data (Fig. 2A and Fig. 3B). Fig. 2A shows that the initial rise of the simulated waveform is faster 
in a $\mathrm{GCAPs}^{-/-}$rod where $\mathrm{Ca}^{2+}$ feedback is absent, which suggests that the main reason for the discrepancy between data and simulations is not related to our assumptions about $\mathrm{Ca}^{2+}$ feedback. A possible reason for this difference could be due to the neglect of multiple phosphorylation steps, which are likely to have a larger impact on rods than cones. Multiple phosphorylations are known to be important for the rod single-photon response (Field \& Rieke, 2002; Hamer et al., 2003; Reingruber \& Holcman, 2008a) and are therefore likely also relevant for dim-flash responses. On the assumption that the intermediate step involving transducin activation proceeds very rapidly, corresponding to a high rate $\mu_{t r}$, the discrepancy between data and model increases because a faster intermediate step accelerates the initial rise of the simulated response (see Fig. 3B). The inclusion of multiple phosphorylation steps would have the opposite effect and would slow down the initial rise of the response, which would lead to a better agreement between data and model.

With our parsimonious model, we are the first to provide a comprehensive mathematical analysis for the whole of the dim-flash response. Our analysis dissects the response dynamics to identify how parameters or parameter combinations govern various phases of the response. This analysis provides a more precise conceptual understanding of how transduction processes determine the dynamics of the photocurrent. We hope that this new insight will lead to the design of new experiments to test these findings in more detail. We hope that this work will serve as a template to derive more refined analytic expressions with more complex models and will help to revitalize the analytic analysis of phototransduction models that was pioneered by Lamb and Pugh (Lamb \& Pugh, 1992; Pugh \& Lamb, 1993). In addition, our approach may also be useful in understanding other G-protein signaling cascades, for example in olfaction (see for example Reisert \& Reingruber, 2019). 
Table 1. Parameter definitions and descriptions.

\begin{tabular}{|l|l|}
\hline Parameter & \\
\hline$\kappa\left(\mu m^{2}\right)$ & Collecting area \\
\hline$n_{c h}$ & CNG channel cooperativity \\
\hline$\beta_{d}\left(s^{-1}\right)$ & Rate constant of cGMP hydrolysis in darkness \\
\hline$\mu_{r h}\left(s^{-1}\right)$ & Rate of deactivation of an activated visual pigment \\
\hline$\mu_{p d e}\left(s^{-1}\right)$ & Rate of deactivation of a light-activated PDE \\
\hline$\mu_{t r}\left(s^{-1}\right)$ & Rate of deactivation of activated transducin \\
\hline$\widehat{K}_{\alpha}$ & $\begin{array}{l}\text { Sensitivity of the cyclase activity on the Ca }{ }^{2+} \text { concentration } \\
\text { scaled by the dark Ca }{ }^{2+} \text { concentration }\end{array}$ \\
\hline$\xi$ & Gain $\xi=\frac{n_{c h} \beta_{s u b} k_{a c t}}{\mu_{p d e} \mu_{r h}}$ \\
\hline$k_{a c t}\left(s^{-1}\right)$ & Rate of transducin activation by an activated visual pigment \\
\hline$\beta_{s u b}\left(s^{-1}\right)$ & Rate constant of cGMP hydrolysis by a light-activated PDE \\
\hline
\end{tabular}


Table 2. Parameter values for the rod and cone models.

\begin{tabular}{|l|c|c|}
\hline Parameter & Rod & Cone \\
\hline$\kappa\left(\mu m^{2}\right)$ & $0.28(\mathrm{WT})$ & 0.013 \\
& $0.42\left(G C A P s^{-/}\right)$ & \\
\hline$n_{c h}$ & 2.5 & 2.5 \\
\hline$\xi$ & 0.45 & 0.0018 \\
\hline$\beta_{d}\left(s^{-1}\right)$ & 4.1 & 11.0 \\
\hline$\mu_{p d e}\left(s^{-1}\right)$ & 5 & 37.8 \\
\hline$\mu_{r h}\left(s^{-1}\right)$ & 28 & 70.7 \\
\hline$\mu_{t r}\left(s^{-1}\right)$ & 23.8 & 70.7 \\
\hline$\widehat{K}_{\alpha}$ & 0.87 & 0.84 \\
\hline
\end{tabular}




\section{FIGURE LEGENDS}

Figure 1. Log-transform of dim-flash responses. Experimental current recordings from mouse rods and cones have been normalized to their corresponding steady-state current in darkness $I_{d}$, with $\hat{I}=\frac{I}{I_{d}}$ (such that $\hat{I}=1$ in darkness), and then the log-transform of these normalized currents was computed according to $y(t)=-\ln \hat{I}(t)$ (black curves). The experimental data is compared to simulations of $y(t)$ (red curves) and the analytic results $y(t)=R_{0}^{*} \xi g_{y}(t)$ computed with Eq. 1 and Eq. 9 and parameters from Table 2. The expected number of pigment isomerizations $R_{0}^{*}=\kappa \phi \Delta t$ are $(1.8,4.3,10.3)$ for $(\mathrm{A}),(0.6,1.6,2.7)$ for $(\mathrm{B})$, and $(86,220,620)$ for (C-D). Flash durations are 10ms in (A), 20ms in (B) and $5 \mathrm{~ms}$ in (C, D). (A) The rod WT data is computed with mean responses of 7 WT rods. (B) The rod $G C A P s^{-/}$data is computed with mean responses of 5 rods from Chen et al. (2010c). (C) The Cone Gnat1 ${ }^{-1-}$ (equivalent to WT or control, see text) data is obtained with responses from 3 cones. (D) The cone Gnat $^{-/} ;$GCAPs $^{-/}$(equivalent to cone GCAPs $^{-/}$, see text) shows mean responses from 3 cones.

Figure 2. Waveform of dim-flash responses. The rod and cone waveforms $\hat{y}(\mathrm{t})=\frac{y(t)}{y_{\text {peak }}}$ from Fig. 1 (thin continuous lines) were used to compute an averaged waveform (thick continuous lines). The averaged waveform is compared to the analytic result $\hat{y}(t)=\hat{g}_{y}(t)$ computed with Eq. 9 and parameters from Table 2 (green lines). (A) Waveforms for rod WT (black curves) and $G C A P s^{-/-}$data (red curves) together with the corresponding analytic results (green curves). (B) Waveforms for cone WT (black curves) and $\mathrm{CCAPS}^{-/}$data (red curves) together with the corresponding analytic results (green curves). 
Figure 3. Rising phase of the waveform of a WT rod. (A) The curves show the first four terms of the Taylor series expansion of $\hat{g}_{y}(t)$ computed with Eq. 9 and WT rod parameters from Table 2. The leading-order term is $\frac{1}{6} \frac{\mu_{r h} \mu_{p d e} \mu_{t r}}{\hat{g}_{y, p e a k}} t^{3}$. (B) The red curve shows the data for the averaged WT rod waveform from Fig. 2A. The green curves show quadratic fits to data and $\hat{g}_{y}(t)$ performed with the fitting function $\frac{1}{2} \hat{A}\left(t-t_{\text {eff }}\right)^{2}$. The fitted values for the waveform amplification constant are $\hat{A} \approx 97 s^{-2}$ (data) and $\hat{A} \approx 135 s^{-2}\left(\hat{g}_{y}\right)$. The blue continuous curve displays $\hat{g}_{y}(t)$ when the parameter $\mu_{t r}$ is assumed to be very large. The leading order term of the Taylor expansion of $\hat{g}_{y}(t)$ for large $\mu_{t r}$ is $\frac{1}{2} \frac{\mu_{r h} \mu_{p d e}}{\hat{g}_{y, p e a k}} t^{2}$ (blue dashed curve).

Figure 4. Recovery phase of the waveform. (A-B) The recovery phase of the averaged waveform data for WT rod and Gnat1 $^{-/-} ; G C A P s^{-/-}$cone from Fig. 2 (black curves) were fitted with a single exponential function to extract the recovery rates constants $\mu_{r e c}$ (red curves). The fitted values are $\mu_{r e c}=4.9 s^{-1}$ for WT rod, and $\mu_{r e c}=11.9^{-1}$ for Gnat $1^{-/-} ; G C A P s^{-/-}$cone. (C-D) The curves show the individual single exponentials related to rhodopsin decay, transducin decay, PDE decay and the cyclase rate constant $\beta$ that contribute to the waveform $\hat{g}_{y}=\hat{g}_{y, r h}+\hat{g}_{y, t r}+\hat{g}_{y, p d e}+$ $\hat{g}_{y, \beta}$ computed with Eq. 9 for a WT $\operatorname{rod}(\mathbf{C})$ and a $G C A P s^{-/-} \operatorname{rod}(\mathbf{D})$.

Figure 5. Estimation of the gain $\xi$ from the peak amplitudes of dim-flash responses. (A) The peak amplitudes $y_{\text {peak }}$ of the log-normal data for rods shown in Fig. 1 A-B together with $g_{y, p e a k}$ 
computed with Eq. 1 and parameters from Table 2 are used to calculate $\frac{y_{\text {peak }}}{g_{y, p e a k}}$ for WT (blue diamonds) and $G C A P s^{-/}$rod (red diamonds), and then plotted against the number of isomerizations $R_{0}^{*}$ specified in Fig. 1. The black curve is a linear fit to the combined datapoints with slope $\xi=0.42$. (B) Same procedure as in (A) but for cones. The linear fit gives $\xi=$ 0.0019 .

Figure 6. Simulations of saturating flash responses for rods and cones. The number of isomerizations $R_{0}^{*}=\kappa \phi \Delta t$ that were used for the simulations are: (A) WT rod $(40,140,600,1200,10000)$, GCAPs $^{-/} \operatorname{rod}(15,50,250,500,4500) ;(\mathrm{B})$ Gnat1 $^{-/}$cone $10^{3} \mathrm{x}(4.55,1.95,45.5,19.5,455)$, Gnat1 $^{-/-} ; G C A P s^{-/-}$cone $10^{3} \mathrm{x}(1.95,9.75,19.5,78,195)$. (A) Comparison of the dynamics of the log-normal $y(t)$ computed with Eq. 1 for saturating flashes for WT (black) and $G C A P s^{-/}$rods (red). Flash intensities for WT and $G C A P s^{-/}$rods are not the same but were chosen to obtain similar peak amplitudes in order to facilitate the comparison of the kinetics. Parameters are from Table 2. (B) Same procedure as in (A) but for cones. (C) The time course of the ratio $r$ of the rod cGMP synthesis to hydrolysis rate (Eq. 14) for the two strongest flashes in (A) is depicted. (D) Same procedure as in (C) but for cone. 


\section{FIGURES}

Figure 1
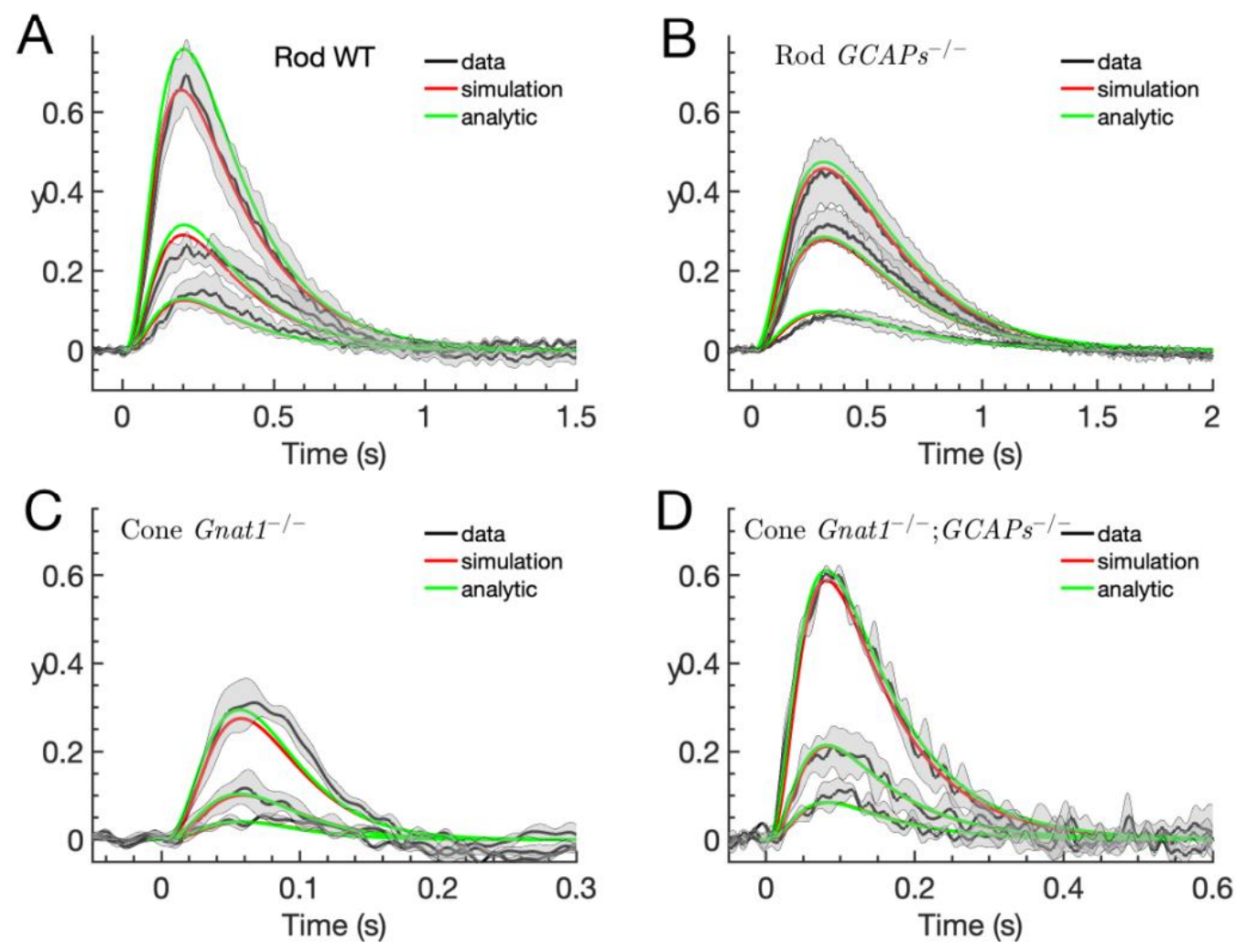

Figure 2
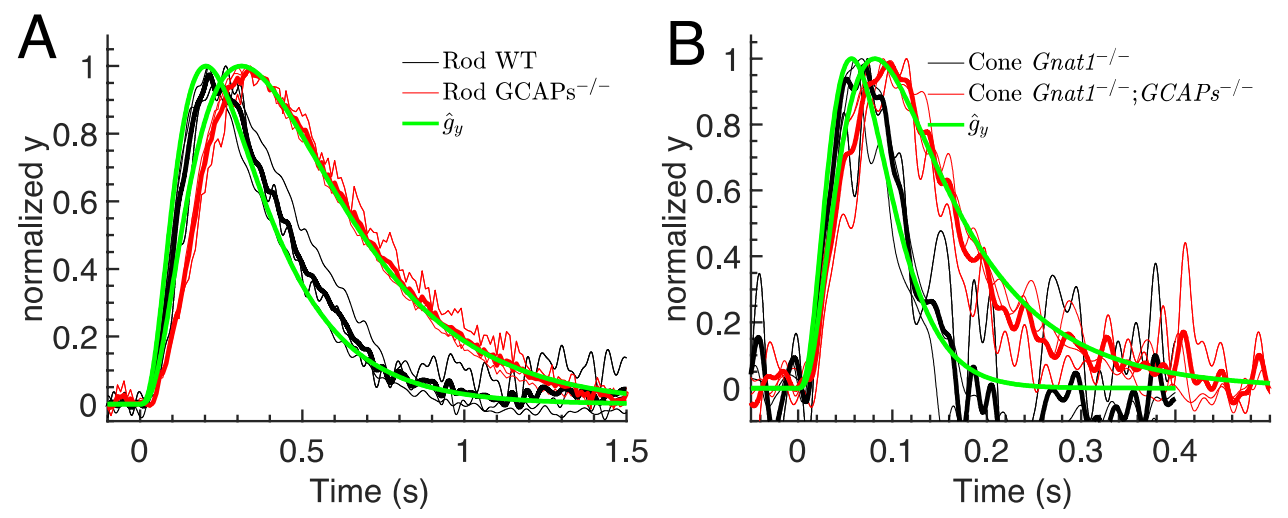

Figure 3 

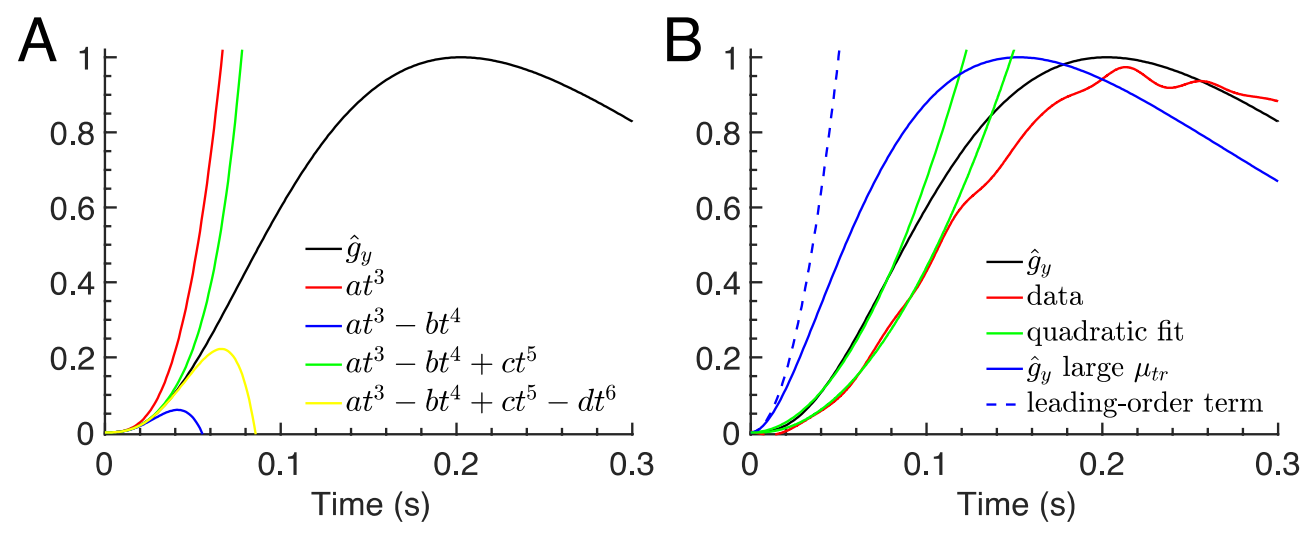

\section{Figure 4}
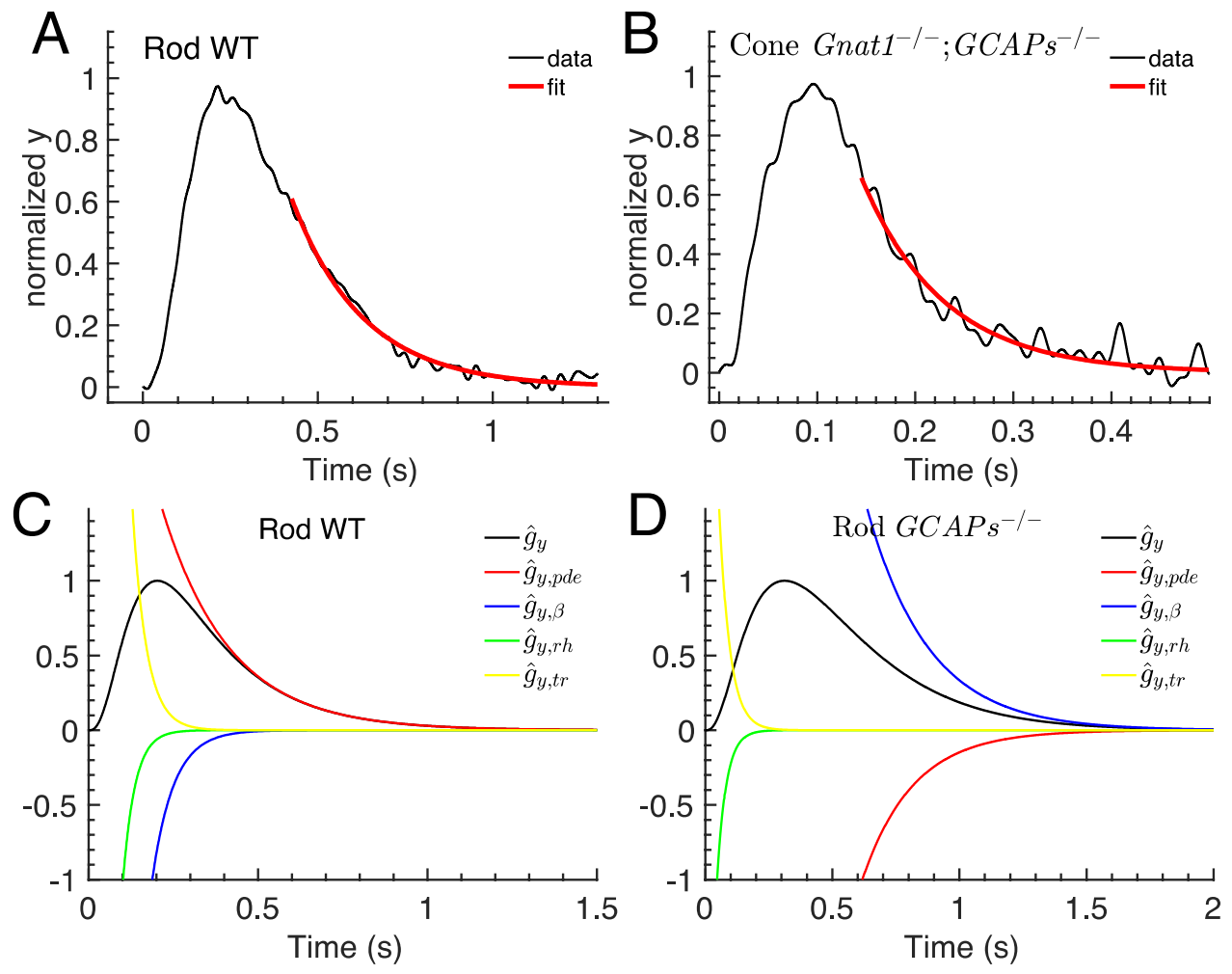

Figure 5 

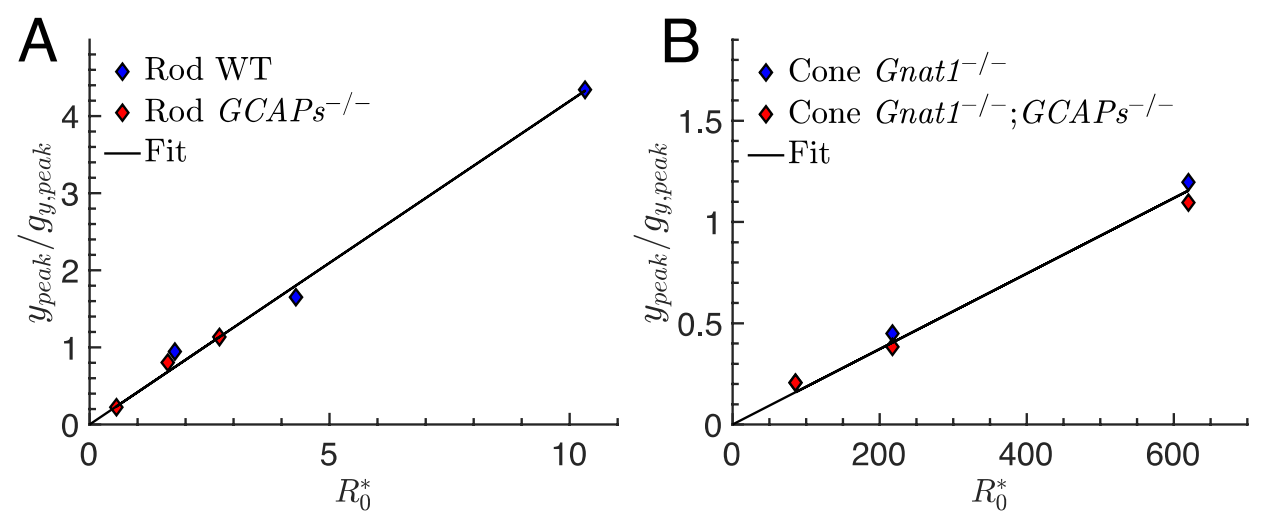

Figure 6
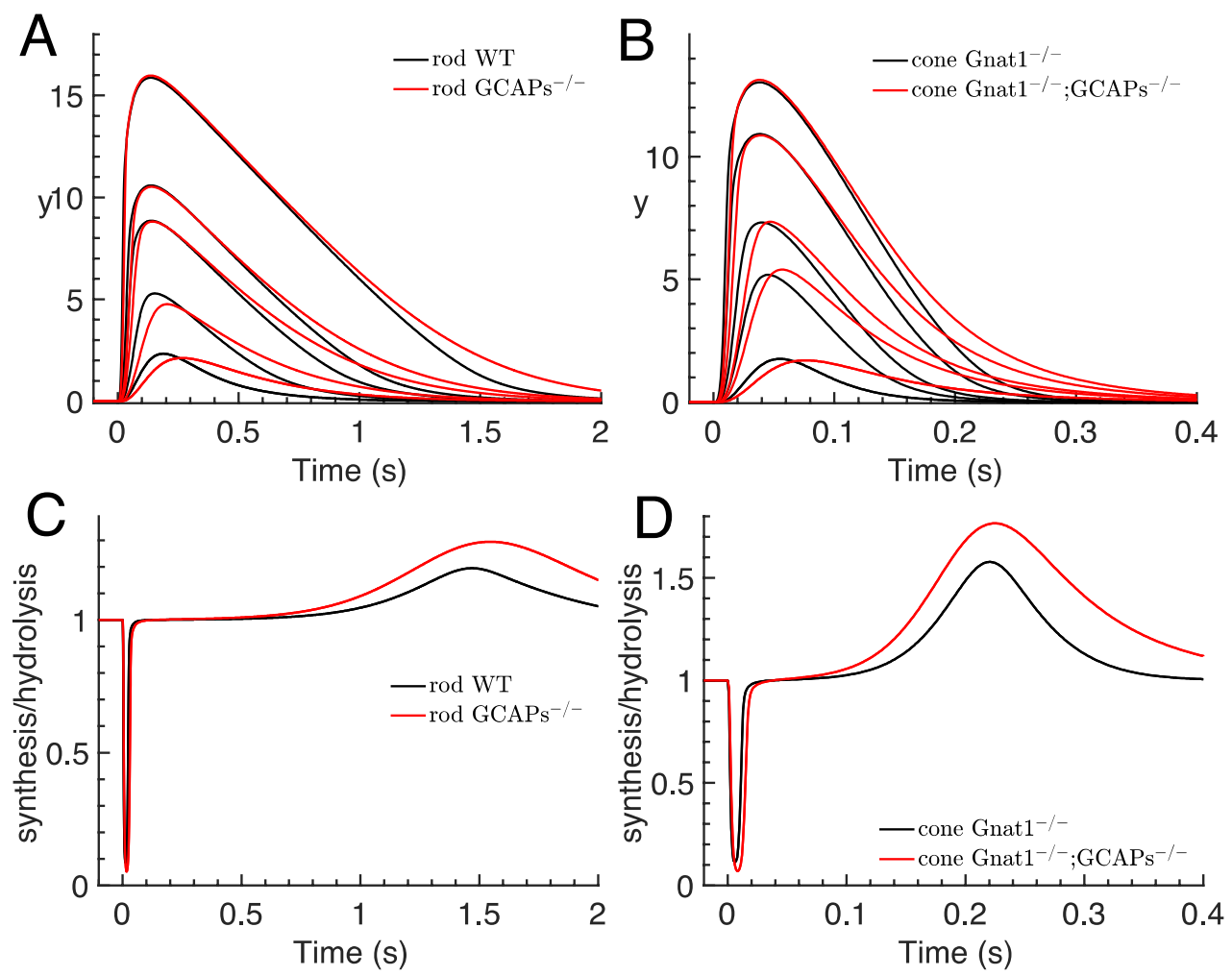


\section{REFERENCES}

Andreucci D, Bisegna P, Caruso G, Hamm HE \& DiBenedetto E. (2003). Mathematical model of the spatio-temporal dynamics of second messengers in visual transduction. Biophys $J \mathbf{8 5}$, 1358-1376.

Arshavsky VY \& Burns ME. (2012). Photoreceptor signaling: supporting vision across a wide range of light intensities. J Biol Chem 287, 1620-1626.

Astakhova L, Firsov M \& Govardovskii V. (2015). Activation and quenching of the phototransduction cascade in retinal cones as inferred from electrophysiology and mathematical modeling. Mol Vis 21, 244-263.

Asteriti S, Gargini C \& Cangiano L. (2017). Connexin 36 expression is required for electrical coupling between mouse rods and cones. Visual Neuroscience 34, e006.

Baylor DA, Hodgkin AL \& Lamb TD. (1974). The electrical response of turtle cones to flashes and steps of light. $J$ Physiol (Lond) 242, 685-727.

Burns ME, Mendez A, Chen J \& Baylor DA. (2002). Dynamics of cyclic GMP synthesis in retinal rods. Neuron 36, 81-91.

Burns ME \& Pugh EN, Jr. (2010). Lessons from photoreceptors: turning off G-protein signaling in living cells. Physiology (Bethesda) 25, 72-84.

Calvert PD, Ho TW, LeFebvre YM \& Arshavsky VY. (1998). Onset of feedback reactions underlying vertebrate rod photoreceptor light adaptation. J Gen Physiol 111, 39-51.

Cameron DA \& Pugh EN, Jr. (1990). The magnitude, time course and spatial distribution of current induced in salamander rods by cyclic guanine nucleotides. $J$ Physiol (Lond) 430, 419-439.

Caruso G, Bisegna P, Andreucci D, Lenoci L, Gurevich VV, Hamm HE \& DiBenedetto E. (2011). Identification of key factors that reduce the variability of the single photon response. Proc Natl Acad Sci U S A 108, 7804-7807.

Caruso G, Bisegna P, Shen L, Andreucci D, Hamm HE \& DiBenedetto E. (2006). Modeling the role of incisures in vertebrate phototransduction. Biophys J 91, 1192-1212. 
Chen CK, Woodruff ML, Chen FS, Chen D \& Fain GL. (2010a). Background light produces a recoverin-dependent modulation of activated-rhodopsin lifetime in mouse rods. $J$ Neurosci 30, 1213-1220.

Chen CK, Woodruff ML, Chen FS, Shim H, Cilluffo MC \& Fain G. (2010b). Replacing the rod with the cone transducin alpha subunit decreases sensitivity and accelerates response decay. J Physiol 588, 3231-3241.

Chen J, Woodruff ML, Wang T, Concepcion F, Tranchina D \& Fain GL. (2010c). Channel modulation and the mechanism of light adaptation in mouse rods. $J$ Neurosci 30, 1623216240.

Dumke CL, Arshavsky VY, Calvert PD, Bownds MD \& Pugh EN, Jr. (1994). Rod outer segment structure influences the apparent kinetic parameters of cyclic GMP phosphodiesterase. $J$ Gen Physiol 103, 1071-1098.

Fain GL. (2019). Sensory Transduction (second edition). Oxford University Press, Oxford.

Field GD \& Rieke F. (2002). Mechanisms regulating variability of the single photon responses of mammalian rod photoreceptors. Neuron 35, 733-747.

Gross OP, Pugh EN, Jr. \& Burns ME. (2012a). Calcium feedback to cGMP synthesis strongly attenuates single-photon responses driven by long rhodopsin lifetimes. Neuron 76, 370382.

Gross OP, Pugh EN, Jr. \& Burns ME. (2012b). Spatiotemporal cGMP dynamics in living mouse rods. Biophys $J$ 102, 1775-1784.

Hamer RD, Nicholas SC, Tranchina D, Lamb TD \& Jarvinen JL. (2005). Toward a unified model of vertebrate rod phototransduction. Vis Neurosci 22, 417-436.

Hamer RD, Nicholas SC, Tranchina D, Liebman PA \& Lamb TD. (2003). Multiple steps of phosphorylation of activated rhodopsin can account for the reproducibility of vertebrate rod single-photon responses. J Gen Physiol 122, 419-444.

Heck M \& Hofmann KP. (2001). Maximal rate and nucleotide dependence of rhodopsincatalyzed transducin activation: initial rate analysis based on a double displacement mechanism. J Biol Chem 276, 10000-10009. 
Holcman D \& Korenbrot JI. (2004). Longitudinal diffusion in retinal rod and cone outer segment cytoplasm: the consequence of cell structure. Biophys $J \mathbf{8 6}, 2566-2582$.

Ingram NT, Fain GL \& Sampath AP. (2020). Elevated Energy Requirement of Cone Photoreceptors. Proc Natl Acad Sci U S A 117, 19599-19603.

Ingram NT, Sampath AP \& Fain GL. (2016). Why are rods more sensitive than cones? Journal of Physiology 594, 5415-5426.

Ingram NT, Sampath AP \& Fain GL. (2019). Voltage-clamp recordings of light responses from wild-type and mutant mouse cone photoreceptors. Journal of General Physiology 151, 1287-1299.

Invergo BM, Dell'Orco D, Montanucci L, Koch KW \& Bertranpetit J. (2014). A comprehensive model of the phototransduction cascade in mouse rod cells. Mol Biosyst 10, 1481-1489.

Jin N, Zhang Z, Keung J, Youn SB, Ishibashi M, Tian L-M, Marshak DW, Solessio E, Umino Y, Fahrenfort I, Kiyama T, Mao E-A, You Y, Wei H, Wu J, Postma F, Paul DL, Massey SC $\&$ Ribelayga CP. (2020). Molecular and functional architecture of the mouse photoreceptor network. Science Advances 6, eaba7232.

Kawamura S \& Tachibanaki S. (2008). Rod and cone photoreceptors: molecular basis of the difference in their physiology. Comp Biochem Physiol A Mol Integr Physiol 150, 369377.

Korenbrot JI. (2012). Speed, sensitivity, and stability of the light response in rod and cone photoreceptors: facts and models. Prog Retin Eye Res 31, 442-466.

Koutalos Y \& Yau KW. (1996). Regulation of sensitivity in vertebrate rod photoreceptors by calcium. Trends Neurosci 19, 73-81.

Krispel CM, Chen D, Melling N, Chen YJ, Martemyanov KA, Quillinan N, Arshavsky VY, Wensel TG, Chen CK \& Burns ME. (2006). RGS Expression Rate-Limits Recovery of Rod Photoresponses. Neuron 51, 409-416.

Lamb TD, Heck M \& Kraft TW. (2018). Implications of dimeric activation of PDE6 for rod phototransduction. Open Biol 8: 180076.

Lamb TD \& Kraft TW. (2020). A quantitative account of mammalian rod phototransduction with PDE6 dimeric activation: responses to bright flashes. Open Biol 10, 190241. 
Lamb TD \& Pugh EN, Jr. (1992). A quantitative account of the activation steps involved in phototransduction in amphibian photoreceptors. J Physiol (Lond) 449, 719-758.

Majumder A, Pahlberg J, Boyd KK, Kerov V, Kolandaivelu S, Ramamurthy V, Sampath AP \& Artemyev NO. (2013). Transducin translocation contributes to rod survival and enhances synaptic transmission from rods to rod bipolar cells. Proc Natl Acad Sci U S A 110: $12468-12473$.

Martemyanov KA, Krispel CM, Lishko PV, Burns ME \& Arshavsky VY. (2008). Functional comparison of RGS9 splice isoforms in a living cell. Proc Natl Acad Sci U S A 105, 20988-20993.

Nikonov S, Lamb TD \& Pugh EN, Jr. (2000). The role of steady phosphodiesterase activity in the kinetics and sensitivity of the light-adapted salamander rod photoresponse. J Gen Physiol 116, 795-824.

Nikonov SS, Kholodenko R, Lem J \& Pugh EN, Jr. (2006). Physiological features of the S- and M-cone photoreceptors of wild-type mice from single-cell recordings. J Gen Physiol 127, 359-374.

Olson A \& Pugh EN, Jr. (1993). Diffusion coefficient of cyclic GMP in salamander rod outer segments estimated with two fluorescent probes. Biophys $J \mathbf{6 5}, 1335-1352$.

Pepperberg DR, Cornwall MC, Kahlert M, Hofmann KP, Jin J, Jones GJ \& Ripps H. (1992). Light-dependent delay in the falling phase of the retinal rod photoresponse. Vis Neurosci 8, 9-18.

Pugh EN, Jr. \& Lamb TD. (1993). Amplification and kinetics of the activation steps in phototransduction. Biochim Biophys Acta 1141, 111-149.

Pugh EN, Jr., Lamb TD (2000) Phototransduction in vertebrate rods and cones: molecular mechanism of amplification, recovery and light adaptation. In: Handbook of Biological Physics: Molecular Mechanisms of Visual Transduction (Stavenga DG, DeGrip WJ, Pugh EN, Jr, eds), pp 183-255. Amsterdam: Elsevier.

Reingruber J \& Holcman D. (2008a). The dynamics of phosphodiesterase activation in rods and cones. Biophys J 94, 1954-1970. 
Reingruber J \& Holcman D. (2008b). Estimating the rate constant of cyclic GMP hydrolysis by activated phosphodiesterase in photoreceptors. Journal of Chemical Physics 129, 145102.

Reingruber J, Holcman D \& Fain GL. (2015). How rods respond to single photons: Key adaptations of a G-protein cascade that enable vision at the physical limit of perception. Bioessays 37, 1243-1252.

Reingruber J, Ingram NT, Griffis C \& Fain GL. (2020). A kinetic analysis of mouse rod and cone photoreceptor responses. Journal of Physiology 598, 3747-3763.

Reingruber J, Pahlberg J, Woodruff ML, Sampath AP, Fain GL \& Holcman D. (2013). Detection of single photons by toad and mouse rods. Proc Natl Acad Sci U S A 110, 19378-19383.

Reisert J \& Reingruber J. (2019). $\mathrm{Ca}^{2+}$-activated $\mathrm{Cl}^{-}$current ensures robust and reliable signal amplification in vertebrate olfactory receptor neurons. Proc Natl Acad Sci U S A 116, 1053-1058.

Rieke F \& Baylor D. (1996). Molecular origin of continuous dark noise in rod photoreceptors. Biophysical Journal 71, 2553-2572.

Rieke F \& Baylor DA. (1998). Origin of reproducibility in the responses of retinal rods to single photons. Biophysical Journal 75, 1836-1857.

Shen L, Caruso G, Bisegna P, Andreucci D, Gurevich VV, Hamm HE \& DiBenedetto E. (2010). Dynamics of mouse rod phototransduction and its sensitivity to variation of key parameters. IET Syst Biol 4, 12-32.

Tranchina D, Sneyd J \& Cadenas ID. (1991). Light adaptation in turtle cones. Testing and analysis of a model for phototransduction. Biophys $J \mathbf{6 0}, 217-237$.

Tsang SH, Woodruff ML, Chen CK, Yamashita CY, Cilluffo MC, Rao AL, Farber DB \& Fain GL. (2006). GAP-Independent termination of photoreceptor light response by excess gamma subunit of the c-GMP-phosphodiesterase. J Neurosci 26, 4472 - 4480.

Vinberg F, Turunen TT, Heikkinen H, Pitkanen M \& Koskelainen A. (2015). A novel Ca ${ }^{2+}$ feedback mechanism extends the operating range of mammalian rods to brighter light. $J$ Gen Physiol 146, 307-321.

Woodruff ML, Rajala A, Fain GL \& Rajala RV. (2014). Modulation of mouse rod photoreceptor responses by Grb14 protein. J Biol Chem 289, 358-364. 